\title{
Vessel Distribution at the Site
}

An essential aspect in the examination of this pottery assemblage is the distribution of vessels inside the graves. An overview of this distribution is presented in Chapter 7.18, where the vessels are presented in their archaeological context (see p. 319, this volume). The distribution of the pottery needs more detailed discussion, and analysis should focus on several aspects, showing the placement of individual vessel types, groups of similar types, and vessel function. Tumuli of types I and II all have multiple chambers, and it is only the smallest tumuli (type III) that do not. It is important to look at the distribu-

\section{Legend to figures and tables:}

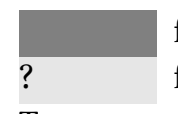

feature not present in given tumulus

Tum. tumulus number

Ch. chamber

E Tun. Eastern tunnel

WTun. Western tunnel

Based on minimum vessel numbers

\section{1}

\section{Distribution by Type}

The distribution of the vessels presented below encompasses only those vessels attributed to particular (identified) types. The author presents the distribution of vessel types inside the tumuli and inside the burial chambers, highlighting the arrangement of particular types.

The distribution shown in this chapter does not include vessels presented in the catalogue as varia or miscella- tion and arrangement of vessels, not only in the context of specific tumulus types, but also in the context of individual chambers within a given tumulus. Can we observe any significant differences in the pottery deposited in particular chambers? All of these issues are discussed by the author in this chapter. The majority of the excavated vessels were discovered complete, almost complete, or were reconstructed from sherds. In those instances where vessels were fragmentary, the statistics presented below were established based on minimum vessel numbers. neous objects. Some examples of pottery rarely noted at the El-Zuma cemetery (goblets [group Ix] and amphorae [type XII.3]) were only recorded by photographing them in situ because the burial chambers in which they were found could not be excavated due to safety concerns. These vessels are, however, included in the calculations presented in this chapter (see also Volume I, Chapter 3, T.6 and T.7). 
Group I-Cups

TABLE 5.1 Cups of type I.1, distribution inside the tumuli

\section{Tumuli type I}

\begin{tabular}{l|c|c|c|c|c|c|c|c}
\hline Tum. & Shaft & Tun. & E Tun. & WTun. & Ch.1 & Ch.2 & Ch.3 & $=$ \\
\hline T.2 & & & & & & 2 & 2 & 4 \\
\hline T.3 & & & & & $?$ & $?$ & $?$ & \\
\hline T.5 & & & & & & & & \\
\hline T.8 & & & & & & $?$ & $?$ & \\
\hline T.1 & & & & & $?$ & $?$ & $?$ & \\
\hline T.4 & & 1 & 1 & 2 & & $?$ & $?$ & 4 \\
\hline T.6 & & & & & $?$ & $?$ & $?$ & \\
\hline T.7 & 2 & & & & $?$ & $?$ & $?$ & 2 \\
\hline Total & & & & & & & & \\
\hline
\end{tabular}

\section{Tumuli type II}

\begin{tabular}{l|c|c|c|c|c|c|c}
\hline Tum. & Shaft & Ch.1 & Ch.2 & Ch.3 & Ch.4 & Ch.5 & $=$ \\
\hline T.12 & & & & & & & \\
\hline T.13 & & & 2 & & & & 2 \\
\hline T.14 & 1 & 2 & 1 & & & & 4 \\
\hline T.15 & 3 & 2 & 2 & & & & 7 \\
\hline T.23 & 1 & 2 & 4 & & & & 7 \\
\hline T.24 & 1 & 2 & & 1 & & & 4 \\
\hline T.25 & & 1 & 1 & 5 & & & 7 \\
\hline T.26 & 2 & 4 & & & & & 6 \\
\hline T.16 & & & & & & & \\
\hline T.9 & & & & & & & \\
\hline T.11 & & & & & & & \\
\hline Total & & & & & & \\
\hline
\end{tabular}

Vessel type $\mathrm{I} .1=50$

PRODUCED BY E. CZYŻEWSKA-ZALEWSKA

\section{Tumuli type III}

\begin{tabular}{l|c|c|c}
\hline Tum. & Shaft & Ch.1 & $=$ \\
\hline T.10 & & 1 & 1 \\
\hline T.17 & & & \\
\hline T.18 & & & \\
\hline T.19 & & & \\
\hline T.20 & 1 & & 1 \\
\hline T.22 & & & \\
\hline T.27 & & & \\
\hline T.21 & 1 & & 1 \\
\hline T.28 & & & \\
\hline Total & & 3 & \\
\hline
\end{tabular}


TABLE 5.2 Cups of type I.2, distribution inside the tumuli

Tumuli type I

\begin{tabular}{l|l|l|l|l|l|c|c|c|c}
\hline Tum. & Mound & Shaft & Tunnel & E Tun. & WTun. & Ch.1 & Ch.2 & Ch.3 & $=$ \\
\hline T.2 & & & & & & & & & \\
\hline T.3 & & & & & & $?$ & $?$ & $?$ & \\
\hline T.5 & 1 & & & & & 1 & 1 & & 3 \\
\hline T.8 & & & & & & $?$ & $?$ & $?$ & \\
\hline T.1 & & & & & & $?$ & $?$ & $?$ & \\
\hline T.4 & & & & & & & $?$ & $?$ & \\
\hline T.6 & & 2 & & & & $?$ & $?$ & $?$ & 2 \\
\hline T.7 & & & & & & $?$ & $?$ & $?$ & \\
\hline Total & & & & & & & & \\
\hline
\end{tabular}

\section{Tumuli type II}

\begin{tabular}{l|c|c|c|c|c|c|c}
\hline Tum. & Shaft & Ch.1 & Ch.2 & Ch.3 & Ch.4 & Ch.5 & $=$ \\
\hline T.12 & & 1 & & 8 & & & 9 \\
\hline T.13 & & & & & & & \\
\hline T.14 & & 1 & & & & & 1 \\
\hline T.15 & & & & & & & \\
\hline T.23 & & & & & & & \\
\hline T.24 & & & & & & & \\
\hline T.25 & & & & & & & \\
\hline T.26 & & & & & & & \\
\hline T.16 & & & & & & & \\
\hline T.9 & & & & & & & \\
\hline T.11 & 1 & 3 & 3 & & & & 7 \\
\hline Total & & & & 17 & & \\
\hline
\end{tabular}

Vessel type $\mathrm{I} .2=35$

PRODUCED BY E. CZYŻEWSKA-ZALEWSKA

\section{Tumuli type III}

\begin{tabular}{|c|c|c|c|}
\hline Tum. & Shaft & Ch.1 & $=$ \\
\hline T.10 & & 5 & 5 \\
\hline \multicolumn{4}{|l|}{ T.17 } \\
\hline T.18 & & 2 & 2 \\
\hline T.19 & 1 & 1 & 2 \\
\hline \multicolumn{4}{|l|}{ T.2O } \\
\hline T.22 & & 1 & 1 \\
\hline \multicolumn{4}{|l|}{ T.27 } \\
\hline \multicolumn{4}{|l|}{ T.21 } \\
\hline T.28 & 1 & 2 & 3 \\
\hline Total & & 13 & \\
\hline
\end{tabular}


TABLE 5.3 Cups of type I.3, distribution inside the tumuli

\begin{tabular}{l|c|c|c|c|c|c|c}
\hline \multicolumn{5}{l}{ Tumuli type II } \\
\hline Tum. & Shaft & Ch.1 & Ch.2 & Ch.3 & Ch.4 & Ch.5 & $=$ \\
\hline T.12 & & & & 3 & & & 3 \\
\hline T.13 & & & & & & & \\
\hline T.14 & & 1 & 1 & 1 & & & 3 \\
\hline T.15 & 1 & & & & & & 1 \\
\hline T.23 & & 4 & 2 & & & & 6 \\
\hline T.24 & & & & & & & \\
\hline T.25 & & & & & & & \\
\hline T.26 & & & & & & & \\
\hline T.16 & & & & & & & \\
\hline T.9 & & & & & & & \\
\hline T.11 & & & & & & & \\
\hline Total & & & & & & & \\
\hline
\end{tabular}

Vessel type I.3 $=19$

PRODUCED BY E. CZYŻEWSKA-ZALEWSKA

TABLE 5.4 Cups of type I.4, distribution inside the tumuli

\section{Tumuli type II}

\begin{tabular}{l|l|l|l|l|l|l|c}
\hline Tum. & Shaft & Ch.1 & Ch.2 & Ch.3 & Ch.4 & Ch.5 & $=$ \\
\hline T.12 & & & & 2 & & & 2 \\
\hline T.13 & & & & & & & \\
\hline T.14 & & & & & & & \\
\hline T.15 & & & & & & & \\
\hline T.23 & & & & & & & \\
\hline T.24 & & & & & & & \\
\hline T.25 & & & & & & & \\
\hline T.26 & & 1 & & & & & \\
\hline T.16 & & & & & & & \\
\hline T.9 & & & & & & & \\
\hline T.11 & & & & & & & \\
\hline Total & & & & & & & \\
\hline
\end{tabular}

Vessel type I.4 $=3$

PRODUCED BY E. CZYŻEWSKA-ZALEWSKA
Tumuli type III

\begin{tabular}{l|c|c|c}
\hline Tum. & Shaft & Ch.1 & $=$ \\
\hline T.10 & & 1 & 1 \\
\hline T.17 & & & \\
\hline T.18 & & & \\
\hline T.19 & & & \\
\hline T.20 & & & \\
\hline T.22 & & 2 & 2 \\
\hline T.27 & & 1 & 1 \\
\hline T.21 & & 1 & 1 \\
\hline T.28 & & 5 & \\
\hline Total & & \multicolumn{3}{|c}{} \\
\hline
\end{tabular}


TABLE 5.5 Cups of type I.5, distribution inside the tumuli

\begin{tabular}{l|l|l|l|l|l|l|l}
\hline \multicolumn{7}{l}{ Tumuli type II } \\
\hline Tum. & Shaft & Ch.1 & Ch.2 & Ch.3 & Ch.4 & Ch.5 & $=$ \\
\hline T.12 & & & & & & & \\
\hline T.13 & & & & & & & \\
\hline T.14 & & & & & & & \\
\hline T.15 & & & & & & & \\
\hline T.23 & & & & & & & \\
\hline T.24 & & & & & & & \\
\hline T.25 & & & & & & & \\
\hline T.26 & & & & & & & \\
\hline T.16 & & & & & & & \\
\hline T.9 & & & & & & & \\
\hline T.11 & & 2 & & & & & 2 \\
\hline Total & & & & 2 & & \\
\hline
\end{tabular}

Vessel type $\mathrm{I} .5=2$

PRODUCED BY E. CZYŻEWSKA-ZALEWSKA

Cups of type I.1 were rare in type I tumuli, and only ten of the fifty type I.1 cups found at El-Zuma were recorded in this category of tumulus. In T.2, which was fully excavated, such cups were discovered in situ in chambers 2 and 3 . In the second fully excavated type I burial, T.5, no such cups were recorded. All other vessels of this type found in type I tumuli were recovered from the tunnel or external shaft (in T.4 and T.7). The most numerous group of thirty-seven type I.1 cups came from type II tumuli. Some examples were recorded in the shafts, but most vessels were found in situ in the main burial chamber and additional chambers. A similar situation to that in tumuli of type I was observed in type III tumuli, where only three such vessels were recorded, all of them either in a shaft or burial chamber.

Cups of type I.2 presented a similar picture. Only five of the thirty-five vessels of this type were discovered in the largest tumuli (type I), three examples being found in T.5 and only two in the external shaft of T.6. In graves of type II, sixteen vessels were recorded in situ, and one in a shaft. They represent half of the assemblage of type I.2 cups, but these vessels came from only three tombs (T.12, T.14 and T.11), so they cannot be regarded as common vessels in this type of tumulus. These cups seem to be most common in type III graves, where thirteen examples were discovered in five tombs, eleven of them in situ.

Cups of type I.3 were only recorded in burials of types II and III. Most of these cups (fourteen of nineteen) were excavated in five graves of type II, and most of them were found in situ in the main chamber and in additional chambers (T.12, T.14, T.23 and T.11). T.15 was the only tomb in which such a vessel was found in the shaft. In type III tumuli only five vessels were found in situ in four graves.

Cups of types I.4 and I.5 occur sporadically. Only three type I.4 vessels were found inside two graves of type II:T.12 and T.26. All examples were found in situ in chambers. Two type $\mathrm{I} .5$ cups were recorded in the main chamber inside T.11 (type II).

Some cups of types I.1-I.4 were present inside the unexcavated burial chambers in T.6 and T.7 (see Volume I, Chapter 3, Fig. 3.35 and Fig. 3.41). This observation was based on photographic records, and it was not possible to establish the exact types represented, but it is, nonetheless, essential to note that such cups were deposited in these type I tumuli.

Generally, cups are abundant in tumuli of type II. 
1.2 Group II-Bowls

1.2.1 II.1-II.7 Small Bowls

TABLE 5.6 Bowls of type II.1, distribution inside the tumuli

\begin{tabular}{|c|c|c|c|c|c|c|c|c|}
\hline \multicolumn{9}{|c|}{ Tumuli type I } \\
\hline Tum. & Shaft & Tun. & E Tun. & WTun. & Ch.1 & Ch.2 & Ch.3 & $=$ \\
\hline \multicolumn{9}{|l|}{ T.2 } \\
\hline T.3 & & & & & $?$ & $?$ & $?$ & \\
\hline \multicolumn{9}{|l|}{$\mathrm{T} .5$} \\
\hline T.8 & & & & & $?$ & $?$ & $?$ & \\
\hline T.1 & & & & & $?$ & $?$ & $?$ & \\
\hline T.4 & & & & & & $?$ & $?$ & \\
\hline T.6 & & 4 & & & $?$ & $?$ & $?$ & 4 \\
\hline T.7 & & & & & $?$ & $?$ & $?$ & \\
\hline Total & & & & & & & & \\
\hline
\end{tabular}

Tumuli type II

\begin{tabular}{l|l|l|l|l|l|l|c}
\hline Tum. & Shaft & Ch.1 & Ch.2 & Ch.3 & Ch.4 & Ch.5 & $=$ \\
\hline T.12 & & & 1 & & & & 1 \\
\hline T.13 & & & & & & & \\
\hline T.14 & & & & & & & \\
\hline T.15 & & & & & & & \\
\hline T.23 & & & & & & & \\
\hline T.24 & & & & & & & \\
\hline T.25 & & & & & & & \\
\hline T.26 & & & & & & & \\
\hline T.16 & & & & & & & \\
\hline T.9 & & & & & & & \\
\hline T.11 & & & 4 & & & & \\
\hline Total & & & & 5 & & & \\
\hline
\end{tabular}

Vessel type II.1 $=14$

PRODUCED BY E. CZYŻEWSKA-ZALEWSKA
Tumuli type III

\begin{tabular}{l|c|c|c|c}
\hline Tum. & Mound & Shaft & Ch.1 & $=$ \\
\hline T.10 & & & 2 & 2 \\
\hline T.17 & & & & \\
\hline T.18 & & & & \\
\hline T.19 & & 1 & 1 & 2 \\
\hline T.20 & & & & \\
\hline T.22 & & & & \\
\hline T.27 & & & & \\
\hline T.21 & 1 & & & 1 \\
\hline T.28 & & & & \\
\hline Total & & & 5 &
\end{tabular}


TABLE 5.7 Bowls of type II.2, distribution inside the tumuli

\begin{tabular}{|c|c|c|c|c|c|c|c|c|}
\hline \multicolumn{9}{|c|}{ Tumuli type I } \\
\hline Tum. & Shaft & Tun. & E Tun. & WTun. & Ch.1 & Ch.2 & Ch.3 & $=$ \\
\hline T.2 & & & & & & 1 & & 1 \\
\hline T.3 & & 1 & & & $?$ & $?$ & $?$ & 1 \\
\hline \multicolumn{9}{|l|}{$\mathrm{T} .5$} \\
\hline T.8 & & & & & 1 & $?$ & $?$ & $\mathbf{1}$ \\
\hline T.1 & & 1 & & & $?$ & $?$ & $?$ & $\mathbf{1}$ \\
\hline T.4 & & 1 & 1 & & $?$ & $?$ & $?$ & 2 \\
\hline T.6 & & & & & $?$ & $?$ & $?$ & \\
\hline $\mathrm{T} .7$ & & & & & $?$ & $?$ & $?$ & \\
\hline Total & \multicolumn{8}{|c|}{6} \\
\hline
\end{tabular}

\section{Tumuli type II}

\begin{tabular}{|c|c|c|c|c|c|c|c|}
\hline Tum. & Shaft & Ch.1 & Ch.2 & Ch.3 & Ch.4 & Ch.5 & $=$ \\
\hline T.12 & 16 & & & 20 & & & 36 \\
\hline T.13 & 1 & & 2 & 4 & 3 & 2 & 12 \\
\hline T.14 & & 1 & 3 & 3 & & & 7 \\
\hline T.15 & 1 & 2 & 4 & & & & 7 \\
\hline T.23 & & 5 & 8 & & & & 13 \\
\hline T.24 & 1 & 4 & & & & & 5 \\
\hline T.25 & & & 5 & 3 & & & 8 \\
\hline T.26 & & 4 & 18 & 1 & 4 & & 27 \\
\hline \multicolumn{8}{|l|}{ T.16 } \\
\hline T.9 & & 1 & & & & & 1 \\
\hline \multicolumn{8}{|l|}{ T.11 } \\
\hline Total & & & & 116 & & & \\
\hline
\end{tabular}

Vessel type II.2 $=127$

PRODUCED BY E. CZYŻEWSKA-ZALEWSKA

\section{Tumuli type III}

\begin{tabular}{l|c|c|c}
\hline Tum. & Shaft & Ch.1 & $=$ \\
\hline T.10 & & 4 & 4 \\
\hline T.17 & & & \\
\hline T.18 & & & \\
\hline T.19 & & & \\
\hline T.20 & & & \\
\hline T.22 & & & \\
\hline T.27 & & & \\
\hline T.21 & 1 & & 1 \\
\hline T.28 & & & \\
\hline Total & & 5 & \\
\hline
\end{tabular}


TABLE 5.8 Bowls of type II.3, distribution inside the tumuli

Tumuli type I

\begin{tabular}{l|l|l|l|l|l|l|l|l}
\hline Tum. & Shaft & Tun. & E Tun. & WTun. & Ch.1 & Ch.2 & Ch.3 & $=$ \\
\hline T.2 & & & & & & & & \\
\hline T.3 & & & & & $?$ & $?$ & $?$ & \\
\hline T.5 & & & & & & & & \\
\hline T.8 & & & & & & $?$ & $?$ & \\
\hline T.1 & & & & & $?$ & $?$ & $?$ & \\
\hline T.4 & & & & & & $?$ & $?$ & \\
\hline T.6 & & 2 & & & $?$ & $?$ & $?$ & 2 \\
\hline T.7 & & & & & $?$ & S & $?$ & \\
\hline Total & & & & & & & \\
\hline
\end{tabular}

\section{Tumuli type II}

\begin{tabular}{l|c|c|c|c|c|c|c}
\hline Tum. & Shaft & Ch.1 & Ch.2 & Ch.3 & Ch.4 & Ch.5 & $=$ \\
\hline T.12 & 11 & & & 1 & & & 12 \\
\hline T.13 & & & & & & & \\
\hline T.14 & & & & & & & \\
\hline T.15 & & 1 & & & & & 1 \\
\hline T.23 & & & & & & & \\
\hline T.24 & & & & & & & \\
\hline T.25 & & & & & & & \\
\hline T.26 & & & & & & & \\
\hline T.16 & & & & & & & \\
\hline T.9 & & & & & & & \\
\hline T.11 & 1 & 4 & 3 & & & & 8 \\
\hline Total & & & & 21 & & \\
\hline
\end{tabular}

Vessel type II.3 $=28$

PRODUCED BY E. CZYŻEWSKA-ZALEWSKA

\section{Tumuli type III}

\begin{tabular}{l|c|c|c}
\hline Tum. & Shaft & Ch.1 & $=$ \\
\hline T.10 & & 4 & 4 \\
\hline T.17 & & & \\
\hline T.18 & & & \\
\hline T.19 & & & \\
\hline T.20 & & & \\
\hline T.22 & & & \\
\hline T.27 & & & \\
\hline T.21 & & 1 & 1 \\
\hline T.28 & & & \\
\hline Total & & 5 & \\
\hline
\end{tabular}


TABLE 5.9 Bowls of type II.4, distribution inside the tumuli

\section{Tumuli type I}

\begin{tabular}{l|c|c|c|c|c|c|c|c}
\hline Tum. & Shaft & Tun. & E Tun. & WTun. & Ch.1 & Ch.2 & Ch.3 & $=$ \\
\hline T.2 & & & & & & 18 & 7 & 25 \\
\hline T.3 & 2 & & & & $?$ & $?$ & $?$ & 2 \\
\hline T.5 & & & & & & & & \\
\hline T.8 & & & & & & $?$ & $?$ & \\
\hline T.1 & 2 & 1 & & & $?$ & $?$ & $?$ & 3 \\
\hline T.4 & & 14 & 7 & & & $?$ & $?$ & 21 \\
\hline T.6 & & & & & $?$ & $?$ & $?$ & \\
\hline T.7 & & 1 & 8 & & $?$ & $?$ & $?$ & 9 \\
\hline Total & & & & 60 & & & \\
\hline
\end{tabular}

\section{Tumuli type II}

\begin{tabular}{l|c|c|c|c|c|c|c}
\hline Tum. & Shaft & Ch.1 & Ch.2 & Ch.3 & Ch.4 & Ch.5 & $=$ \\
\hline T.12 & 1 & & & & & & 1 \\
\hline T.13 & & & 1 & 1 & & & 2 \\
\hline T.14 & & & 1 & & & 1 \\
\hline T.15 & & & & & & & \\
\hline T.23 & & & 5 & & & \\
\hline T.24 & & & & & & & \\
\hline T.25 & & & & & & & \\
\hline T.26 & & & 1 & & & & 1 \\
\hline T.16 & & & & & & & \\
\hline T.9 & 3 & & & & & & 3 \\
\hline T.11 & & & & & & & \\
\hline Total & & & & 13 & & \\
\hline
\end{tabular}

Vessel type II.4 = 73

PRODUCED BY E. CZYŻEWSKA-ZALEWSKA 
TABLE 5.10 Bowls of type II.5, distribution inside the tumuli

\begin{tabular}{l|l|l|l|l|l|l|l}
\hline \multicolumn{5}{l}{ Tumuli type II } & \multicolumn{5}{l}{} \\
\hline Tum. & Shaft & Ch.1 & Ch.2 & Ch.3 & Ch.4 & Ch.5 & $=$ \\
\hline T.12 & & & & & & & \\
\hline T.13 & & & & & & & \\
\hline T.14 & & & & & & & \\
\hline T.15 & 2 & 2 & & & & & 4 \\
\hline T.23 & & & & & & & \\
\hline T.24 & & & & & & & \\
\hline T.25 & & & & & & & \\
\hline T.26 & & & & & & & \\
\hline T.16 & & & & & & & \\
\hline T.9 & & & & & & & \\
\hline T.11 & & & & & & & \\
\hline Total & & & & & & & \\
\hline
\end{tabular}

Vessel type II.5 = 4

PRODUCED BY E. CZYŻEWSKA-ZALEWSKA

Fourteen bowls of type II.1 were recorded at the cemetery. Four of them were found in the tunnel of T.6 (type I), five examples were recorded in situ in T.12 and T.11 (type II), and a further five vessels were found in three tumuli of the smallest type (III): two in the burial chamber in T.10 and one in the shaft, and one in the burial chamber in T.19.

A different situation was observed with bowls of type II.2. Seven of these vessels were found in the largest, type I, tumuli: six in the tunnels of T.3, T.1, and T.4, and one in chamber 1 in T.8. Single vessels were also recorded in type III tumuli: four in the burial chamber of T.1o and one in the shaft of T.21. In type II tumuli these bowls were much more numerous. One hundred and sixteen bowls were discovered in these tombs, examples being noted in every tumulus except T.11 and T.16. Most of this assemblage was found in situ. Only sixteen bowls were recorded as one group in the shaft of T.12. Single examples were found in the shafts of T.13, T.15, and T.24. This is the most numerous type of small bowl at the El-Zuma cemetery.

Twenty-eight bowls of type II.3 were discovered, and their distribution is similar to that of type II.2 bowls. In the largest graves (type I) there were only two such vessels, both found in the T.6 tunnel. In tumuli of type III, five examples were found in situ in two tumuli (T.10 and T.21), and a further twenty-one vessels were recorded in T.12, T.15, and T.11. Eleven vessels were discovered as one large group in the shaft of T.12 (see Volume I, Chapter 3).

Bowls of type II.4 were only found in graves of types I and II. Most of these vessels (sixty of seventy-three) were recorded in type I tumuli: twenty-five of them were found in situ in T.2, while thirty-five (mostly incomplete or fragmentary) were recovered from the tunnels or external shafts in T.3, T.1, T.4 and T.7. Thirteen vessels were found in six tumuli of type II, mainly in situ.

Bowls of types II.1-3 and II.4 were recorded in situ in the chamber of T.7, which was unexcavated because of safety concerns (see Volume I, Chapter 3, Fig. 3.41).

Type II. 5 bowls are represented by four vessels discovered in one type II tumulus (T.15): two were found in the shaft and two in the main chamber.

Type II.6 bowls are also represented by four vessels. One fragmentarily preserved bowl was recovered from the mound of T.7, and its original context is uncertain, but the other three bowls were discovered in the chambers of T.13 and T.14.

Type II.7 is the last group of small bowls and is also represented by four vessels. Two of them were discovered in the external shaft of type I tumulus T.6, and the other two examples were found in chamber 2 of T.11 (type II). 
TABLE 5.11 Bowls of type II.6, distribution inside the tumuli

Tumuli type I

\begin{tabular}{l|l|l|l|l|l|c|c|c|c}
\hline Tum. & Mound & Shaft & Tun. & E Tun. & WTun. & Ch.1 & Ch.2 & Ch.3 & $=$ \\
\hline T.2 & & & & & & & & & \\
\hline T.3 & & & & & & $?$ & $?$ & $?$ & \\
\hline T.5 & & & & & & & & & \\
\hline T.8 & & & & & & & $?$ & $?$ & \\
\hline T.1 & & & & & & $?$ & $?$ & $?$ & \\
\hline T.4 & & & & & & & $?$ & $?$ & \\
\hline T.6 & & & & & & $?$ & $?$ & $?$ & \\
\hline T.7 & 1 & & & & & $?$ & $?$ & $?$ & 1 \\
\hline Total & & & & 1 & & & & \\
\hline
\end{tabular}

\section{Tumuli type II}

\begin{tabular}{l|l|l|l|l|l|l|l}
\hline Tum. & Shaft & Ch.1 & Ch.2 & Ch.3 & Ch.4 & Ch.5 & $=$ \\
\hline T.12 & & & & & & & \\
\hline T.13 & & & & & 1 & & 1 \\
\hline T.14 & & & & 2 & & & 2 \\
\hline T.15 & & & & & & & \\
\hline T.23 & & & & & & & \\
\hline T.24 & & & & & & & \\
\hline T.25 & & & & & & & \\
\hline T.26 & & & & & & & \\
\hline T.16 & & & & & & & \\
\hline T.9 & & & & & & & \\
\hline T.11 & & & & & & & \\
\hline Total & & & & 3 & & & \\
\hline
\end{tabular}

Vessel type II. $6=4$

PRODUCED BY E. CZYŻEWSKA-ZALEWSKA

Bowls of types II.1-II.3 are wheel-made, red-slipped, and burnished. They were quite common in tumuli types II and III and were mainly found in situ. In type I tumuli, such bowls were found sporadically and mainly in the external shafts and tunnels. Wheel-made, slipped, and polished bowls of type II.4, with grooved decoration, were abundant in graves of type I, and only a small number were found in situ in the side chambers of four tumuli of type II. Of the seventy-three type II.4 vessels, sixty were discovered in type I tumuli and only thirteen in type II tumuli. Bowls of types II.5, II.6, and II.7 occur sporadically and their number is too small for meaningful analysis. 
TABLE 5.12 Bowls of type II.7, distribution inside the tumuli

Tumuli type I

\begin{tabular}{l|l|l|l|l|l|l|l|l}
\hline Tum. & Shaft & Tun. & E Tun. & WTun. & Ch.1 & Ch.2 & Ch.3 & $=$ \\
\hline T.2 & & & & & & & & \\
\hline T.3 & & & & & $?$ & $?$ & $?$ & \\
\hline T.5 & & & & & & & & \\
\hline T.8 & & & & & & $?$ & $?$ & \\
\hline T.1 & & & & & $?$ & $?$ & $?$ & \\
\hline T.4 & & & & & & $?$ & $?$ & \\
\hline T.6 & 2 & & & & $?$ & $?$ & $?$ & 2 \\
\hline T.7 & & & & & $?$ & $?$ & $?$ & \\
\hline Total & & & & & & & \\
\hline
\end{tabular}

\section{Tumuli type II}

\begin{tabular}{l|l|l|l|l|l|l|l}
\hline Tum. & Shaft & Ch.1 & Ch.2 & Ch.3 & Ch.4 & Ch.5 & $=$ \\
\hline T.12 & & & & & & & \\
\hline T.13 & & & & & & & \\
\hline T.14 & & & & & & & \\
\hline T.15 & & & & & & & \\
\hline T.23 & & & & & & & \\
\hline T.24 & & & & & & & \\
\hline T.25 & & & & & & & \\
\hline T.26 & & & & & & & \\
\hline T.16 & & & & & & & \\
\hline T.9 & & & & & & & \\
\hline T.11 & & & 2 & & & & 2 \\
\hline Total & & & & 2 & & & \\
\hline
\end{tabular}

Vessel type II.7 $=4$

based on minimal vessel number

PRODUCED BY E. CZYŻEWSKA-ZALEWSKA 
1.2.2 II.8-II.9 Large Bowls

TABLE 5.13 Bowls of type II.8, distribution inside the tumuli

\section{Tumuli type I}

\begin{tabular}{|c|c|c|c|c|c|c|c|c|}
\hline Tum. & Shaft & Tun. & E Tun. & W Tun. & Ch.1 & Ch.2 & Ch.3 & $=$ \\
\hline \multicolumn{9}{|l|}{ T.2 } \\
\hline $\mathrm{T} \cdot 3$ & & 1 & & & $?$ & $?$ & $?$ & $\mathbf{1}$ \\
\hline $\mathrm{T} .5$ & 1 & & & & & & & 1 \\
\hline T.8 & & 1 & & & & $?$ & $?$ & $\mathbf{1}$ \\
\hline T.1 & & & & & ? & $?$ & $?$ & \\
\hline T.4 & & 1 & & & & $?$ & $?$ & 1 \\
\hline T.6 & & 2 & & & ? & $?$ & ? & 2 \\
\hline T.7 & & 1 & & & ? & $?$ & ? & $\mathbf{1}$ \\
\hline Total & \multicolumn{8}{|c|}{7} \\
\hline
\end{tabular}

\section{Tumuli type II}

\begin{tabular}{l|c|c|c|c|c|c|c}
\hline Tum. & Shaft & Ch.1 & Ch.2 & Ch.3 & Ch.4 & Ch.5 & $=$ \\
\hline T.12 & & & & & & & \\
\hline T.13 & & & & & & & \\
\hline T.14 & & & & & & & \\
\hline T.15 & & & & & & & \\
\hline T.23 & & & & & & & \\
\hline T.24 & 1 & & & & & & 1 \\
\hline T.25 & & & & & & & \\
\hline T.26 & 1 & & & & & & 1 \\
\hline T.16 & & & & & & & \\
\hline T.9 & & & & & & & \\
\hline T.11 & & & & & & & \\
\hline Total & & & & & & & \\
\hline Vesset & & & & & & & \\
\hline
\end{tabular}

Vessel type II. $8=10$

PRODUCED BY E. CZYŻEWSKA-ZALEWSKA
Tumuli type III

\begin{tabular}{l|l|l|l|l}
\hline Tum. & Mound & Shaft & Ch.1 & $=$ \\
\hline T.10 & & & & \\
\hline T.17 & & & & \\
\hline T.18 & & & & \\
\hline T.19 & & & & \\
\hline T.20 & & & & \\
\hline T.22 & & & & \\
\hline T.27 & & & & \\
\hline T.21 & 1 & & & 1 \\
\hline T.28 & & & & \\
\hline Total & & & 1 & \\
\hline
\end{tabular}


TABLE 5.14 Bowls of type II.9, distribution inside the tumuli

Tumuli type I

\begin{tabular}{l|c|c|c|c|c|c|c|c}
\hline Tum. & Shaft & Tun. & E Tun. & WTun. & Ch.1 & Ch.2 & Ch.3 & $=$ \\
\hline T.2 & & & & & & & & \\
\hline T.3 & & & & & $?$ & $?$ & $?$ & \\
\hline T.5 & & & & & & & & \\
\hline T.8 & & & & & & $?$ & $?$ & \\
\hline T.1 & 1 & & & & $?$ & $?$ & $?$ & 1 \\
\hline T.4 & & & & & & $?$ & $?$ & \\
\hline T.6 & & 1 & & & $?$ & $?$ & $?$ & 1 \\
\hline T.7 & & 1 & & & $?$ & $?$ & $?$ & 1 \\
\hline Total & & & & 3 & & & \\
\hline
\end{tabular}

\section{Tumuli type II}

\begin{tabular}{l|l|l|l|l|l|l|l}
\hline Tum. & Shaft & Ch.1 & Ch.2 & Ch.3 & Ch.4 & Ch.5 & $=$ \\
\hline T.12 & & & & & & & \\
\hline T.13 & & & & & & & \\
\hline T.14 & & & & & & & \\
\hline T.15 & & & & & & & \\
\hline T.23 & & & & & & & \\
\hline T.24 & 0.5 & & 0.5 & & & & 1 \\
\hline T.25 & & & & & & & \\
\hline T.26 & & & & & & & \\
\hline T.16 & & & & & & & \\
\hline T.9 & & & & & & & \\
\hline T.11 & & & 1 & & & & 1 \\
\hline Total & & & & 2 & & \\
\hline
\end{tabular}

Vessel type II.9 $=5$

PRODUCED BY E. CZYŻEWSKA-ZALEWSKA

Large bowls of types II.8 and II.9 were represented by numerous sherds. Only one large bowl was found in situ (in T.11) and was complete. The rest of the fragmented vessels were discovered mainly in the tunnels, shafts, or superstructures of the graves, and most of them had been used as scrapers (see below, Chapter 5.2). 


\subsection{Group III-Spouted Bowls}

TABLE 5.15 Bowls of types III.1 and III.2, distribution inside the tumuli

\begin{tabular}{|c|c|c|c|c|c|c|c|c|}
\hline \multicolumn{9}{|c|}{ Tumuli type I } \\
\hline Tum. & Shaft & Tun. & E Tun. & WTun. & Ch.1 & Ch.2 & Ch.3 & $=$ \\
\hline \multicolumn{9}{|l|}{ T.2 } \\
\hline $\mathrm{T} .3$ & & & & & $?$ & $?$ & $?$ & \\
\hline \multicolumn{9}{|l|}{$\mathrm{T} .5$} \\
\hline Т.8 & & & & & & $?$ & $?$ & \\
\hline T.1 & & & & & $?$ & $?$ & $?$ & \\
\hline $\mathrm{T} .4$ & & & & & & $?$ & $?$ & \\
\hline T.6 & & & & & $?$ & $?$ & $?$ & \\
\hline T.7 & 1 & & & & $?$ & $?$ & $?$ & $\mathbf{1}$ \\
\hline Total & & & & & & & & \\
\hline
\end{tabular}

Vessel type $\mathrm{III} .1=3$

Tumuli type II

\begin{tabular}{l|l|l|l|l|l|l|l}
\hline Tum. & Shaft & Ch.1 & Ch.2 & Ch.3 & Ch.4 & Ch.5 & $=$ \\
\hline T.12 & & & & & & & \\
\hline T.13 & & & & & & & \\
\hline T.14 & & & & & & & \\
\hline T.15 & & & & & & & \\
\hline T.23 & & & & & & & \\
\hline T.24 & & 1 & & & & & \\
\hline T.25 & & & & & & & \\
\hline T.26 & & & & & & & \\
\hline T.16 & & & & & & & \\
\hline T.9 & & & & & & & \\
\hline T.11 & & & & & & & \\
\hline Total & & & & & & & \\
\hline
\end{tabular}

Tumuli type II I

\begin{tabular}{l|c|c|c}
\hline Tum. & Shaft & Ch.1 & $=$ \\
\hline T.10 & & & \\
\hline T.17 & & & \\
\hline T.18 & & & \\
\hline T.19 & 1 & & 1 \\
\hline T.20 & & & \\
\hline T.22 & & & \\
\hline T.27 & & & \\
\hline T.21 & & & \\
\hline T.28 & & & \\
\hline Total & & 1 & \\
\hline
\end{tabular}

PRODUCED BY E. CZYŻEWSKA-ZALEWSKA 
TABLE 5.15 Bowls of types III.1 and III.2, distribution inside the tumuli (cont.)

Tumuli type I

\begin{tabular}{l|l|l|l|l|l|l|l|l}
\hline Tum. & Shaft & Tun. & E Tun. & WTun. & Ch.1 & Ch.2 & Ch.3 & $=$ \\
\hline T.2 & & & & & & & & \\
\hline T.3 & & & & & $?$ & $?$ & $?$ & \\
\hline T.5 & & & & & & & & \\
\hline T.8 & & & & & & $?$ & $?$ & \\
\hline T.1 & & & & & $?$ & $?$ & $?$ & \\
\hline T.4 & & & & & & $?$ & $?$ & \\
\hline T.6 & 1 & & & & $?$ & $?$ & $?$ & 1 \\
\hline T.7 & & & & & $?$ & $?$ & $?$ & \\
\hline Total & & & & & & & \\
\hline
\end{tabular}

Vessel type III.2 = 1

Four bowls were recorded at this site. Two almost complete examples, restored from sherds Z19/4, Z24/25 and spout fragment $Z_{7} / 17$, represent handmade bowls of type III.1, while Z6/13 is a complete wheel-made bowl of type
III.2. Only one vessel, Z24/25, was found in situ in chamber 1 of T.24. The rest of these vessels were recorded in the shafts. Their small number is insufficient for broader analysis. 


\subsection{Group IV-Bowls}

TABLE 5.16 Bowls of type IV.1, distribution inside the tumuli

\section{Tumuli type II}

\begin{tabular}{l|l|l|l|l|l|l|l}
\hline Tum. & Shaft & Ch.1 & Ch.2 & Ch.3 & Ch.4 & Ch.5 & $=$ \\
\hline T.12 & & & & & & & \\
\hline T.13 & & & & & & & \\
\hline T.14 & & & & & & & \\
\hline T.15 & & & & & & & \\
\hline T.23 & & & & & & & \\
\hline T.24 & & & & & & & \\
\hline T.25 & & & & & & & \\
\hline T.26 & & & & & & & \\
\hline T.16 & & & & & & & \\
\hline T.9 & & & & & & & \\
\hline T.11 & 2 & & & & & & 2 \\
\hline Total & & & & 2 & & & \\
\hline
\end{tabular}

Vessel type IV.1 $=4$

PRODUCED BY E. CZYŻEWSKA-ZALEWSKA

TABLE 5.17 Bowls of type IV.2, distribution inside the tumuli

\section{Tumuli type II}

\begin{tabular}{l|l|l|l|l|l|l|l}
\hline Tum. & Shaft & Ch.1 & Ch.2 & Ch.3 & Ch.4 & Ch.5 & $=$ \\
\hline T.12 & & & & & & & \\
\hline T.13 & & & & & & & \\
\hline T.14 & & & & & & & \\
\hline T.15 & & & & & & & \\
\hline T.23 & & & & & & & \\
\hline T.24 & & 1 & & & & & 1 \\
\hline T.25 & & & 3 & 1 & & & 4 \\
\hline T.26 & & & & & & & \\
\hline T.16 & & & & & & & \\
\hline T.9 & & & & & & & \\
\hline T.11 & & & & & & & \\
\hline Total & & & & 5 & & \\
\hline
\end{tabular}

Vessel type IV.2 $=5$

PRODUCED BY E. CZYŻEWSKA-ZALEWSKA

\begin{tabular}{l|c|c|c}
\hline \multicolumn{4}{l}{ Tumuli type III } \\
\hline Tum. & Shaft & Ch.1 & $=$ \\
\hline T.10 & & 1 & 1 \\
\hline T.17 & & & \\
\hline T.18 & & 1 & 1 \\
\hline T.19 & & & \\
\hline T.2O & & & \\
\hline T.22 & & & \\
\hline T.27 & & & \\
\hline T.21 & & & \\
\hline T.28 & & & \\
\hline Total & & 2 & \\
\hline
\end{tabular}


TABLE 5.18 Bowls of type IV.3, distribution inside the tumuli

\begin{tabular}{l|l|l|l|l|l|l|l}
\hline \multicolumn{5}{l}{ Tumuli type II } & \multicolumn{5}{l}{} \\
\hline Tum. & Shaft & Ch.1 & Ch.2 & Ch.3 & Ch.4 & Ch.5 & $=$ \\
\hline T.12 & & & & & & & \\
\hline T.13 & & & & & & & \\
\hline T.14 & & & & & & & \\
\hline T.15 & & & & & & & \\
\hline T.23 & & & 1 & & & & 1 \\
\hline T.24 & & & & & & & \\
\hline T.25 & & & & & & & \\
\hline T.26 & & & 3 & & & & \\
\hline T.16 & 1 & & & & & & \\
\hline T.9 & & & & & & & \\
\hline T.11 & & & & & & & \\
\hline Total & & & & & & & \\
\hline
\end{tabular}

Vessel type IV.3 $=8$

PRODUCED BY E. CZYŻEWSKA-ZALEWSKA

TABLE 5.19 Bowls of type IV.4, distribution inside the tumuli

\section{Tumuli type II}

\begin{tabular}{l|l|l|l|l|l|l|c}
\hline Tum. & Shaft & Ch.1 & Ch.2 & Ch.3 & Ch.4 & Ch.5 & $=$ \\
\hline T.12 & & & & 1 & & & 1 \\
\hline T.13 & & & & & & & \\
\hline T.14 & & & & & & & \\
\hline T.15 & & & & & & & \\
\hline T.23 & & & & & & & \\
\hline T.24 & & & & & & & \\
\hline T.25 & & & & & & & \\
\hline T.26 & & & 1 & & & & \\
\hline T.16 & & & & & & & \\
\hline T.9 & & & & & & & \\
\hline T.11 & & & & & & & \\
\hline Total & & & & & & & \\
\hline
\end{tabular}

Vessel type IV.4 $=3$

PRODUCED BY E. CZYŻEWSKA-ZALEWSKA

\begin{tabular}{l|l|l|l}
\hline \multicolumn{3}{l}{ Tumuli type III } & \multicolumn{1}{l}{$=$} \\
\hline Tum. & Shaft & Ch.1 & $=$ \\
\hline T.10 & & & \\
\hline T.17 & & & \\
\hline T.18 & & & \\
\hline T.19 & & & \\
\hline T.20 & & & \\
\hline T.22 & & & \\
\hline T.27 & & & \\
\hline T.21 & & & \\
\hline T.28 & & 1 & 1 \\
\hline Total & & 1 & \\
\hline
\end{tabular}


The numbers of bowls classified as group IV (handmade bowls with clay added to the bottom) are insufficient for any robust analysis. Four complete bowls of type IV.1 were found, two of them in the shaft of T.11 (type II), and another two in the chambers of T.10 and T.18 (type III). Five bowls of type IV.2 were found in the chambers in T.24 and T.25 (type II). Bowls of type IV.3, eight in total, were only recorded in type II tumuli: in T.23 and T.26 (in the cham- bers), and in T.16 (one example was found in the shaft). There were three examples of type IV.4 bowls: two were discovered in T.12 and T.26 (type II), and one in T.28 (type III). Such vessels were also present in the largest graves (type I). In the main burial chamber of T.6 (unexcavated because of safety concerns) a shallow bowl was recognized from the photographic record (see Volume I, Chapter 3, Fig. 3.35).

\subsection{Group V-Pots}

TABLE 5.20 Pots of type V.1, distribution inside the tumuli

\section{Tumuli type II}

\begin{tabular}{l|l|l|l|l|l|l|l}
\hline Tum. & Shaft & Ch.1 & Ch.2 & Ch.3 & Ch.4 & Ch.5 & $=$ \\
\hline T.12 & & & & & & & \\
\hline T.13 & & & & & & & \\
\hline T.14 & & & & & & & \\
\hline T.15 & & & & & & & \\
\hline T.23 & & & & & & & \\
\hline T.24 & & & & & & & \\
\hline T.25 & & & & & & & \\
\hline T.26 & & & & & & & \\
\hline T.16 & & & & & & & \\
\hline T.9 & & & & & & & \\
\hline T.11 & 1 & & & & & & \\
\hline Total & & & & & & & \\
\hline
\end{tabular}

Vessel type V.1 = 4

PRODUCED BY E. CZYŻEWSKA-ZALEWSKA

A similar situation to that of the type IV bowls exists with the group v pots. The small size of this group rules out any meaningful analysis. Type V.1 is represented by four vessels: two were found in T.11 (type II), one in T.10, and one in T.18 (type III). Type V.2 was noted only in type II tumuli: two examples came from T.24, one from T.25, and another

\begin{tabular}{l|c|c|c}
\hline \multicolumn{3}{l}{ Tumuli type III } \\
\hline Tum. & Shaft & Ch.1 & $=$ \\
\hline T.10 & & 1 & 1 \\
\hline T.17 & & & \\
\hline T.18 & 1 & & 1 \\
\hline T.19 & & & \\
\hline T.20 & & & \\
\hline T.22 & & & \\
\hline T.27 & & & \\
\hline T.21 & & & \\
\hline T.28 & & & \\
\hline Total & & 2 & \\
\hline
\end{tabular}

one from T.26. Types V.3 and V.4 were more numerously represented. Ten bowls of type V.3 were recorded in situ in graves of type II (T.14, T.25, T.26, T.9, and T.11). There were also ten type $\mathrm{V} .4$ vessels; nine of them were found in situ in burials of type II (T.12, T.24, T.26), and one was recorded in the burial chamber of T.28 (type III). 
TABLE 5.21 Pots of type V.2, distribution inside the tumuli

Tumuli type II

\begin{tabular}{l|l|l|l|l|l|l|l}
\hline Tum. & Shaft & Ch.1 & Ch.2 & Ch.3 & Ch.4 & Ch.5 & $=$ \\
\hline T.12 & & & & & & & \\
\hline T.13 & & & & & & & \\
\hline T.14 & & & & & & & \\
\hline T.15 & & & & & & & \\
\hline T.23 & & & & & & & \\
\hline T.24 & & 2 & & & & & 2 \\
\hline T.25 & & & & 1 & & & 1 \\
\hline T.26 & & & & 1 & & & \\
\hline T.16 & & & & & & & \\
\hline T.9 & & & & & & & \\
\hline T.11 & & & & & & & \\
\hline Total & & & & & & & \\
\hline Vesstype & & & & & & & \\
\hline
\end{tabular}

Vessel type V.2 $=4$

PRODUCED BY E. CZYŻEWSKA-ZALEWSKA

TABLE 5.22 Pots of type v.3, distribution inside the tumuli

\section{Tumuli type II}

\begin{tabular}{|c|c|c|c|c|c|c|c|}
\hline Tum. & Shaft & Ch.1 & Ch.2 & Ch.3 & Ch.4 & Ch.5 & $=$ \\
\hline \multicolumn{8}{|l|}{ T.12 } \\
\hline \multicolumn{8}{|l|}{ T.13 } \\
\hline T.14 & & 1 & & & & & 1 \\
\hline \multicolumn{8}{|l|}{ T.15 } \\
\hline T.23 & 1 & & & & & & $\mathbf{1}$ \\
\hline \multicolumn{8}{|l|}{ T.24 } \\
\hline T.25 & & & 1 & 2 & & & 3 \\
\hline T.26 & & 1 & 2 & & & & 3 \\
\hline \multicolumn{8}{|l|}{ T.16 } \\
\hline T.9 & & 1 & & & & & 1 \\
\hline T.11 & & 1 & & & & & 1 \\
\hline Total & & & & 10 & & & \\
\hline
\end{tabular}

Vessel type $\mathrm{V} \cdot 3=10$

PRODUCED BY E. CZYŻEWSKA-ZALEWSKA 
TABLE 5.23 Pots of type V.4, distribution inside the tumuli

\begin{tabular}{l|l|l|l|l|l|l|l}
\hline Tumuli type II & \multicolumn{5}{l}{} \\
\hline Tum. & Shaft & Ch.1 & Ch.2 & Ch.3 & Ch.4 & Ch.5 & $=$ \\
\hline T.12 & & & & 1 & & & 1 \\
\hline T.13 & & & & & & & \\
\hline T.14 & & & & & & & \\
\hline T.15 & & & & & & & \\
\hline T.23 & & & & & & & \\
\hline T.24 & & 1 & & & & & 1 \\
\hline T.25 & & & & & & & \\
\hline T.26 & & 3 & & 1 & 2 & & 7 \\
\hline T.16 & & & & & & & \\
\hline T.9 & & & & & & & \\
\hline T.11 & & & & & & & \\
\hline Total & & & & & & & \\
\hline
\end{tabular}

\section{Tumuli type III}

\begin{tabular}{l|c|c|c}
\hline Tum. & Shaft & Ch.1 & $=$ \\
\hline T.10 & & & \\
\hline T.17 & & & \\
\hline T.18 & & & \\
\hline T.19 & & & \\
\hline T.20 & & & \\
\hline T.22 & & & \\
\hline T.27 & & & \\
\hline T.21 & & & \\
\hline T.28 & & 1 & 1 \\
\hline Total & & 1 & \\
\hline
\end{tabular}

Vessel type V.4 $=10$

PRODUCED BY E. CZYŻEWSKA-ZALEWSKA 


\subsection{Group VI-Pots}

TABLE 5.24 Pots of type VI..1, distribution inside the tumuli

\begin{tabular}{|c|c|c|c|c|c|c|c|c|}
\hline \multicolumn{9}{|c|}{ Tumuli type I } \\
\hline Tum. & Shaft & Tun. & E Tun. & WTun. & Ch.1 & Ch.2 & Ch.3 & $=$ \\
\hline \multicolumn{9}{|l|}{ T.2 } \\
\hline T.3 & & & & & $?$ & $?$ & $?$ & \\
\hline $\mathrm{T} .5$ & & 1 & & & & & & $\mathbf{1}$ \\
\hline Т.8 & & & & & & $?$ & $?$ & \\
\hline T.1 & & & & & $?$ & $?$ & $?$ & \\
\hline $\mathrm{T} .4$ & & & & & & $?$ & $?$ & \\
\hline T.6 & & & & & $?$ & $?$ & $?$ & \\
\hline T.7 & & & & & $?$ & $?$ & $?$ & \\
\hline Total & & & & & & & & \\
\hline
\end{tabular}

Tumuli type II

\begin{tabular}{l|l|l|l|l|l|l|l}
\hline Tum. & Shaft & Ch.1 & Ch.2 & Ch.3 & Ch.4 & Ch.5 & $=$ \\
\hline T.12 & & & & & & & \\
\hline T.13 & & & & & & & \\
\hline T.14 & & & & & & & \\
\hline T.15 & & & & & & & \\
\hline T.23 & & & & & & & \\
\hline T.24 & & & & & & & \\
\hline T.25 & & & & & & & \\
\hline T.26 & & & & & & & \\
\hline T.16 & & & & & & & \\
\hline T.9 & & & & & & & \\
\hline T.11 & 2 & & & & & & \\
\hline Total & & & & & & & \\
\hline
\end{tabular}

Vessel type VI.1 $=3$

PRODUCED BY E. CZYŻEWSKA-ZALEWSKA 
TABLE 5.25 Pots of type VI.2, distribution inside the tumuli

\begin{tabular}{l|l|l|c|c|c|c|c|c|c}
\hline Tumuli type I \\
\hline Tum. & Mound & Shaft & Tun. & E Tun. & W Tun. & Ch.1 & Ch.2 & Ch.3 & = \\
\hline T.2 & & & & & & & & \\
\hline T.3 & & & & & & $?$ & $?$ & $?$ & \\
\hline T.5 & & & & & & & & & \\
\hline T.8 & & & & & & & $?$ & $?$ & \\
\hline T.1 & & & & & & $?$ & $?$ & $?$ & \\
\hline T.4 & & & & & & & $?$ & $?$ & \\
\hline T.6 & & & & & & $?$ & $?$ & $?$ & \\
\hline T.7 & 1 & & & & & $?$ & $?$ & $?$ & 1 \\
\hline Total & & & & & 1 & & & & \\
\hline
\end{tabular}

\begin{tabular}{l|l|l|l|l|l|l|l}
\hline Tumuli type II & \multicolumn{5}{l}{} \\
\hline Tum. & Shaft & Ch.1 & Ch.2 & Ch.3 & Ch.4 & Ch.5 & $=$ \\
\hline T.12 & & & & & & & \\
\hline T.13 & & & & & & & \\
\hline T.14 & & & & & & & \\
\hline T.15 & & & & & & & \\
\hline T.23 & & & & & & & \\
\hline T.24 & & & & & & & \\
\hline T.25 & & & & & & & \\
\hline T.26 & & & & & & & \\
\hline T.16 & & & & & & & \\
\hline T.9 & & & & & & & \\
\hline T.11 & 1 & & & & & & \\
\hline Total & & & & & & & \\
\hline
\end{tabular}

Vessel type VI.2 $=3$

PRODUCED BY E. CZYŻEWSKA-ZALEWSKA

\section{Tumuli type III}

\begin{tabular}{l|l|l|l|l}
\hline Tum. & Mound & Shaft & Ch.1 & $=$ \\
\hline T.10 & & & & \\
\hline T.17 & & & & \\
\hline T.18 & & & & \\
\hline T.19 & & & & \\
\hline T.20 & & & & \\
\hline T.22 & & & & \\
\hline T.27 & 1 & & & 1 \\
\hline T.21 & & & & \\
\hline T.28 & & & & \\
\hline Total & & & & \\
\hline
\end{tabular}


TABLE 5.26 Pots of type VI.3, distribution inside the tumuli

\section{Tumuli type II}

\begin{tabular}{l|l|l|l|l|l|l|l}
\hline Tum. & Shaft & Ch.1 & Ch.2 & Ch.3 & Ch.4 & Ch.5 & $=$ \\
\hline T.12 & & & & & & & \\
\hline T.13 & & & & & & & \\
\hline T.14 & & & & & & & \\
\hline T.15 & & & & & & \\
\hline T.23 & & & & & & \\
\hline T.24 & & & & & & \\
\hline T.25 & & & & & & & \\
\hline T.26 & & & & & & & \\
\hline T.16 & & & & & & & \\
\hline T.9 & & & & & & & \\
\hline T.11 & 1 & & & & & & 1 \\
\hline Total & & & & 1 & & \\
\hline
\end{tabular}

Vessel type VI.3 = 1

PRODUCED BY E. CZYŻEWSKA-ZALEWSKA

Six fragmentarily preserved vessels were attributed to this group. Type VI.1 was represented by three vessels, one of which was found in the tunnel of T.5 (type I), while sherds from a further two vessels were recovered from the shaft of T.11 (type II). In type VI.2 one example was discovered in the mound of T.7 (its original context is uncertain), one in the shaft of T.11 and one in the superstructure of T.27. One pot of type VI.3 was found in the shaft of T.11.
Not all of the recorded examples were found in situ. Some were discovered in the shaft fill, in the tunnel, or the tumulus superstructure. The only type in tumulus that yielded group VI sherds was T.11 (all of them recovered from the shaft). 
1.7 Group VII-Jars and Bottles

TABLE 5.27 Jars of type VII.1, distribution inside the tumuli

Tumuli type II

\begin{tabular}{l|c|c|c|c|c|c|c}
\hline Tum. & Shaft & Ch.1 & Ch.2 & Ch.3 & Ch.4 & Ch.5 & $=$ \\
\hline T.12 & 2 & 3 & 1 & & & & 6 \\
\hline T.13 & & & & & & & \\
\hline T.14 & & & & & & & \\
\hline T.15 & & & & & & & \\
\hline T.23 & & & & & & & \\
\hline T.24 & & & & 1 & & & 1 \\
\hline T.25 & & & & & & & \\
\hline T.26 & & & & & & & \\
\hline T.16 & & & & & & & \\
\hline T.9 & & & & 1 & & & 1 \\
\hline T.11 & & & & & & & \\
\hline Total & & & & & & \\
\hline
\end{tabular}

Vessel type VII.1 $=15$

PRODUCED BY E. CZYŻEWSKA-ZALEWSKA

The group viI pottery is divided into six types of vessels. Fifteen examples of type viI.1 jars were recorded in tumuli of types II and III: eight of them were discovered in T.12, T.24, and T.9 (type II), and seven in T.18, T.20, and T.28 (type III). Jars of type VII.2 are represented by twentyone examples. More than half of them were recorded in graves of type II: ten examples were found in T.12, and one apiece in T.13, T.15, and T.25. Seven vessels were discovered in five modestly equipped tumuli of type III, and one jar was discovered in the main burial chamber of T.2, a type I tumulus. Jars of type VII.3 are represented by one specimen found in chamber 3 of T.2. Type VII. 4 is represented by twelve vessels. Four of them were discovered in T.2 and T.5 (type I), and eight of them were recovered from T.12, T.14, T.16, and T.11 (type II). Bottles of types VII.5 and VII.6 are the most numerously represented at this site. Twenty-five were recorded in total, examples being found in each type of tomb, most abundantly in type II tumuli. Twenty vessels or fragments of type viI.5 bottles were recorded, featuring in almost all tumuli of type II, where twelve examples were found in the chambers and eight in the burial shafts. Only two such vessels were excavated in T.2 (type I), and three examples came from the burial chambers of T.10 and T.22 (type III). Nineteen type viI.6 bottles were discovered in seven tumuli of type II: eight were found in the shaft, one in the superstructure, and ten in the burial chambers. Only one jar was noted in a type I tumulus, namely the fully excavated T.5. In type III tumuli, five examples were discovered in situ. 
TABLE 5.28 Jars of type VII.2, distribution inside the tumuli

\begin{tabular}{l|c|c|c|c|c|c|c|c}
\hline \multicolumn{7}{l}{ Tumuli type I } \\
\hline Tum. & Shaft & Tun. & E Tun. & W Tun. & Ch.1 & Ch.2 & Ch.3 & $=$ \\
\hline T.2 & & & & & 1 & & & 1 \\
\hline T.3 & & & & & $?$ & $?$ & $?$ & \\
\hline T.5 & & & & & & & & \\
\hline T.8 & & & & & & $?$ & $?$ & \\
\hline T.1 & & & & & $?$ & $?$ & $?$ & \\
\hline T.4 & & & & & & $?$ & $?$ & \\
\hline T.6 & & & & & $?$ & $?$ & $?$ & \\
\hline T.7 & & & & & $?$ & $?$ & $?$ & \\
\hline Total & & & & & & & \\
\hline
\end{tabular}

\section{Tumuli type II}

\begin{tabular}{l|c|c|c|c|c|c|c}
\hline Tum. & Shaft & Ch.1 & Ch.2 & Ch.3 & Ch.4 & Ch.5 & $=$ \\
\hline T.12 & 7 & 2 & & 1 & & & 10 \\
\hline T.13 & & & 1 & & & & 1 \\
\hline T.14 & & & & & & & \\
\hline T.15 & & 1 & & & & & 1 \\
\hline T.23 & & & & & & & \\
\hline T.24 & & & & & 1 & & 1 \\
\hline T.25 & & & & & & & \\
\hline T.26 & & & & & & & \\
\hline T.16 & & & & & & & \\
\hline T.9 & & & & & & & \\
\hline T.11 & & & & & & & \\
\hline Total & & & & & & \\
\hline
\end{tabular}

Vessel type VII.2 $=21$

PRODUCED BY E. CZYŻEWSKA-ZALEWSKA

\section{Tumuli type III}

\begin{tabular}{l|c|c|c}
\hline Tum. & Shaft & Ch.1 & $=$ \\
\hline T.10 & & 2 & 2 \\
\hline T.17 & & 2 & 2 \\
\hline T.18 & & 1 & 1 \\
\hline T.19 & & & \\
\hline T.20 & & & \\
\hline T.22 & & 1 & 1 \\
\hline T.27 & & & \\
\hline T.21 & & 1 & 1 \\
\hline T.28 & & 7 & \\
\hline Total & & 7 \\
\hline
\end{tabular}


TABLE 5.29 Jars of type VII.3, distribution inside the tumuli

Tumuli type I

\begin{tabular}{l|l|l|l|l|l|l|l|l}
\hline Tum. & Shaft & Tun. & E Tun. & WTun. & Ch.1 & Ch.2 & Ch.3 & $=$ \\
\hline T.2 & & & & & & & 1 & 1 \\
\hline T.3 & & & & & $?$ & $?$ & $?$ & \\
\hline T.5 & & & & & & & & \\
\hline T.8 & & & & & & $?$ & $?$ & \\
\hline T.1 & & & & & $?$ & $?$ & $?$ & \\
\hline T.4 & & & & & & $?$ & $?$ & \\
\hline T.6 & & & & & $?$ & $?$ & $?$ & \\
\hline T.7 & & & & & $?$ & $?$ & $?$ & \\
\hline Total & & & & & & & & \\
\hline
\end{tabular}

Vessel type VII.3 $=1$

PRODUCED BY E. CZYŻEWSKA-ZALEWSKA 
TABLE 5.30 Bottles of type VII.4, distribution inside the tumuli

Tumuli type I

\begin{tabular}{l|l|l|l|l|l|c|c|c|c}
\hline Tum. & Mound & Shaft & Tun. & E Tun. & WTun. & Ch.1 & Ch.2 & Ch.3 & $=$ \\
\hline T.2 & & & & & & & 1 & 2 & 3 \\
\hline T.3 & & & & & & $?$ & $?$ & $?$ & \\
\hline T.5 & & & & & & & 1 & & 1 \\
\hline T.8 & & & & & & & $?$ & $?$ & \\
\hline T.1 & & & & & & $?$ & $?$ & $?$ & \\
\hline T.4 & & & & & & & $?$ & $?$ & \\
\hline T.6 & & & & & & $?$ & $?$ & $?$ & \\
\hline T.7 & & & & & & $?$ & $?$ & $?$ & \\
\hline Total & & & & 4 & & & & \\
\hline
\end{tabular}

\section{Tumuli type II}

\begin{tabular}{l|c|c|c|c|c|c|c|c}
\hline Tum. & Mound & Shaft & Ch.1 & Ch.2 & Ch.3 & Ch.4 & Ch.5 & $=$ \\
\hline T.12 & & 1 & & & & & & 1 \\
\hline T.13 & & & & & & & & \\
\hline T.14 & & & & & 1 & & & 1 \\
\hline T.15 & & 1 & 2 & 1 & & & & 4 \\
\hline T.23 & & & & & & & & \\
\hline T.24 & & & & & & & & \\
\hline T.25 & & & & & & & & \\
\hline T.26 & & & & & & & & \\
\hline T.16 & & & 1 & & & & & 1 \\
\hline T.9 & & & & & & & & \\
\hline T.11 & 1 & & & & & & & 1 \\
\hline Total & & & & & & & & \\
\hline
\end{tabular}

Vessel type VII.4 $=12$

PRODUCED BY E. CZYŻEWSKA-ZALEWSKA 
TABLE 5.31 Bottles of type VII.5, distribution inside the tumuli

\begin{tabular}{l|c|c|c|c|c|c|c|c}
\hline \multicolumn{7}{l}{ Tumuli type I } \\
\hline Tum. & Shaft & Tun. & E Tun. & W Tun. & Ch.1 & Ch.2 & Ch.3 & $=$ \\
\hline T.2 & & & & & & 2 & & 2 \\
\hline T.3 & & & & & $?$ & $?$ & $?$ & \\
\hline T.5 & & & & & & & & \\
\hline T.8 & & & & & & $?$ & $?$ & \\
\hline T.1 & & & & & $?$ & $?$ & $?$ & \\
\hline T.4 & & & & & & $?$ & $?$ & \\
\hline T.6 & & & & & $?$ & $?$ & $?$ & \\
\hline T.7 & & & & & $?$ & $?$ & $?$ & \\
\hline Total & & & & & & & \\
\hline
\end{tabular}

\section{Tumuli type II}

\begin{tabular}{l|c|c|c|c|c|c|c}
\hline Tum. & Shaft & Ch.1 & Ch.2 & Ch.3 & Ch.4 & Ch.5 & $=$ \\
\hline T.12 & 1 & 1 & 1 & & & & 3 \\
\hline T.13 & 2 & & 1 & & & & 3 \\
\hline T.14 & 2 & & & & & & 2 \\
\hline T.15 & & 2 & 1 & & & & 3 \\
\hline T.23 & & & & & & & \\
\hline T.24 & 2 & 2 & & & & & 4 \\
\hline T.25 & & & 2 & & & & 2 \\
\hline T.26 & & & & 1 & & & 1 \\
\hline T.16 & & 1 & & & & & 1 \\
\hline T.9 & & & & & & & \\
\hline T.11 & 1 & & & & & & 1 \\
\hline Total & & & & & & \\
\hline
\end{tabular}

Vessel type VII.5 $=25$

PRODUCED BY E. CZYŻEWSKA-ZALEWSKA

\section{Tumuli type III}

\begin{tabular}{l|c|c|c}
\hline Tum. & Shaft & Ch.1 & $=$ \\
\hline T.10 & & 2 & 2 \\
\hline T.17 & & & \\
\hline T.18 & & & \\
\hline T.19 & & & \\
\hline T.20 & & & \\
\hline T.22 & & 1 & 1 \\
\hline T.27 & & & \\
\hline T.21 & & & \\
\hline T.28 & & & \\
\hline Total & & 3 & \\
\hline
\end{tabular}


TABLE 5.32 Bottles of type VII.6, distribution inside the tumuli

\section{Tumuli type I}

\begin{tabular}{l|c|c|c|c|c|c|c|c}
\hline Tum. & Shaft & Tun. & E Tun. & W Tun. & Ch.1 & Ch.2 & Ch.3 & = \\
\hline T.2 & & & & & & & & \\
\hline T.3 & & & & & $?$ & $?$ & $?$ & \\
\hline T.5 & & & & & 1 & & & 1 \\
\hline T.8 & & & & & & $?$ & $?$ & \\
\hline T.1 & & & & & $?$ & $?$ & $?$ & \\
\hline T.4 & & & & & & $?$ & $?$ & \\
\hline T.6 & & & & & $?$ & $?$ & $?$ & \\
\hline T.7 & & & & & $?$ & $?$ & $?$ & \\
\hline Total & & & & & & & & \\
\hline
\end{tabular}

\section{Tumuli type II}

\begin{tabular}{|c|c|c|c|c|c|c|c|c|}
\hline Tum. & Mound & Shaft & Ch.1 & Ch.2 & Ch.3 & Ch.4 & Ch. 5 & $=$ \\
\hline T.12 & & 6 & 2 & 1 & 2 & & & 11 \\
\hline T.13 & & & & 1 & & & & 1 \\
\hline T.14 & 1 & & & & & & & $\mathbf{1}$ \\
\hline \multicolumn{9}{|l|}{ T.15 } \\
\hline \multicolumn{9}{|l|}{ T.23 } \\
\hline T.24 & & 1 & & & & & & 1 \\
\hline \multicolumn{9}{|l|}{ T.25 } \\
\hline T.26 & & 1 & & & 1 & & & 2 \\
\hline \multicolumn{9}{|l|}{ T.16 } \\
\hline T.9 & & & & 2 & & & & 2 \\
\hline T.11 & & & & 1 & & & & 1 \\
\hline Total & & & & & & & & \\
\hline
\end{tabular}

Vessel type VII. $6=25$

PRODUCED BY E. CZYŻEWSKA-ZALEWSKA

\section{Tumuli type III}

\begin{tabular}{l|c|c|c}
\hline Tum. & Shaft & Ch.1 & $=$ \\
\hline T.10 & & 1 & 1 \\
\hline T.17 & & & \\
\hline T.18 & & 1 & 1 \\
\hline T.19 & & & \\
\hline T.20 & & 2 & 2 \\
\hline T.22 & & & \\
\hline T.27 & & 1 & 1 \\
\hline T.21 & & & \\
\hline T.28 & & & \\
\hline Total & & 5 \\
\hline
\end{tabular}




\subsection{Groups VIII-Stands and IX-Goblets}

TABLE 5.33 Stands of types VIII.1 and VIII.2, distribution inside the tumuli

\begin{tabular}{|c|c|c|c|c|c|c|c|c|}
\hline \multicolumn{9}{|c|}{ Tumuli type I } \\
\hline Tum. & Shaft & Tun. & E Tun. & WTun. & Ch.1 & Ch.2 & Ch.3 & $=$ \\
\hline \multicolumn{9}{|l|}{ T.2 } \\
\hline $\mathrm{T} .3$ & & & & & $?$ & $?$ & $?$ & \\
\hline \multicolumn{9}{|l|}{$\mathrm{T} .5$} \\
\hline Т.8 & & & & & & $?$ & $?$ & \\
\hline T.1 & & & & & $?$ & $?$ & $?$ & \\
\hline T.4 & & & & & & $?$ & $?$ & \\
\hline T.6 & & & & & 1 & $?$ & $?$ & $\mathbf{1}$ \\
\hline T.7 & & 1 & & & $?$ & $?$ & $?$ & $\mathbf{1}$ \\
\hline Total & & & & & & & & \\
\hline
\end{tabular}

Tumuli type II

\begin{tabular}{l|l|l|l|l|l|l|l}
\hline Tum. & Shaft & Ch.1 & Ch.2 & Ch.3 & Ch.4 & Ch.5 & $=$ \\
\hline T.12 & & & & & & & \\
\hline T.13 & & & & & & & \\
\hline T.14 & & & & & & & \\
\hline T.15 & & & & & & & \\
\hline T.23 & & 1 & & & & & \\
\hline T.24 & & & & & & & \\
\hline T.25 & & & & & & & \\
\hline T.26 & & & & & & & \\
\hline T.16 & & & & & & & \\
\hline T.9 & & & & & & & \\
\hline T.11 & & & & & & & \\
\hline Total & & & & & & & \\
\hline
\end{tabular}

Vessel type VIII $=3$

PRODUCED BY E. CZYŻEWSKA-ZALEWSKA 
TABLE 5.34 Goblets of types IX.1, IX.2 and IX.3, distribution inside the tumuli

\begin{tabular}{l|c|c|c|c|c|c|c|c}
\hline \multicolumn{7}{l}{ Tumuli type I } \\
\hline Tum. & Shaft & Tun. & E Tun. & W Tun. & Ch.1 & Ch.2 & Ch.3 & $=$ \\
\hline T.2 & & & & & & & & \\
\hline T.3 & & & & & $?$ & $?$ & $?$ & \\
\hline T.5 & & & & & & & & \\
\hline T.8 & & & & & & $?$ & $?$ & \\
\hline T.1 & & & & & $?$ & $?$ & $?$ & \\
\hline T.4 & & 1 & & & & $?$ & $?$ & 1 \\
\hline T.6 & & & & & $?$ & $?$ & $?$ & \\
\hline T.7 & & & & & 1 & $?$ & $?$ & 1 \\
\hline Total & & & & & & & \\
\hline
\end{tabular}

\section{Tumuli type II}

\begin{tabular}{l|l|l|l|l|l|l|l}
\hline Tum. & Shaft & Ch.1 & Ch.2 & Ch.3 & Ch.4 & Ch.5 & $=$ \\
\hline T.12 & & & & & & & \\
\hline T.13 & & & & & & & \\
\hline T.14 & & & & & & & \\
\hline T.15 & & & & & & & \\
\hline T.23 & & & & & & & \\
\hline T.24 & & 1 & & & & & 1 \\
\hline T.25 & & & & & & & \\
\hline T.26 & & & & & & & \\
\hline T.16 & & & & & & & \\
\hline T.9 & & & & & & & \\
\hline T.11 & & & & & & & \\
\hline Total & & & & 1 & & \\
\hline
\end{tabular}

Vessel type IX $=4$

PRODUCED BY E. CZYŻEWSKA-ZALEWSKA

\section{Tumuli type III}

\begin{tabular}{l|c|c|c}
\hline Tum. & Shaft & Ch.1 & $=$ \\
\hline T.10 & & 1 & 1 \\
\hline T.17 & & & \\
\hline T.18 & & & \\
\hline T.19 & & & \\
\hline T.20 & & & \\
\hline T.22 & & & \\
\hline T.27 & & & \\
\hline T.21 & & & \\
\hline T.28 & & & \\
\hline Total & & 1 & \\
\hline
\end{tabular}


These two groups of vessels are represented by very few examples, and broader analysis of either type is not possible. Only one complete stand was discovered in T.23 (type II), and another fragmented example was found in the tunnel of T.7. A third stand was noted in the main burial chamber of T.6. Although this tomb was not excavated because of safety concerns, the pot stand can be seen in photographs, next to the blocking wall (see Volume I, Chapter 3, Fig. 3.34).

\subsection{Group $x$-Oil Lamps}

Three examples of goblets were found, one in each type of tumulus. A goblet of type IX.1 was recorded in T.24 (type II), a goblet of type IX.2 in T.1o (type III) and a goblet of type IX.3 in the tunnel of T.4 (type I). Another goblet of completely different shape and form was recorded in the unexcavated chamber of T.7 (type I), on the blocking wall (bottom course) of the chamber (see Volume I, Chapter 3 , Fig. 3.41).

TABLE 5.35 Oil lamps of type $\mathrm{X}$, distribution inside the tumuli

\begin{tabular}{l|c|c|c|c|c|c|c|c}
\hline \multicolumn{7}{l}{ Type I tumuli } \\
\hline Tum. & Shaft & Tun. & E Tun. & WTun. & Ch.1 & Ch.2 & Ch.3 & $=$ \\
\hline T.2 & & & & & & & \\
\hline T.3 & & & & $?$ & $?$ & $?$ & \\
\hline T.5 & & & & & & & & \\
\hline T.8 & & & & & & $?$ & $?$ & \\
\hline T.1 & & & & & $?$ & $?$ & $?$ & \\
\hline T.4 & & 1 & 1 & 1 & & $?$ & $?$ & 3 \\
\hline T.6 & & & 1 & & $?$ & $?$ & $?$ & 1 \\
\hline T.7 & & & 1 & & $?$ & $?$ & $?$ & 1 \\
\hline Total & & & & & & & & \\
\hline
\end{tabular}

Vessel type $\mathrm{x}=5$

PRODUCED BY E. CZYŻEWSKA-ZALEWSKA

Five oil lamps were found in three tumuli of type I: three lamps in T.4, one in T.6, and one in T.7. The lamps from $\mathrm{T} .4$ were found in the fill of the $\mathrm{E}$ and $\mathrm{W}$ tunnels, while lamps from another two tombs were found at the top of the E tunnel fill and are probably connected with robber activity (see Volume I, Chapter 3, Fig. 3.34; Chapter 3.6, this volume). 
Group XI-Qawadis

TABLE 5.36 Qawadis of type XI, distribution inside the tumuli

\begin{tabular}{l|c|c|c|c|c|c|c|c}
\hline \multicolumn{7}{l}{ Tumuli type I } \\
\hline Tum. & Shaft & Tun. & E Tun. & WTun. & Ch.1 & Ch.2 & Ch.3 & = \\
\hline T.2 & & & & & & & \\
\hline T.3 & & 1 & & & $?$ & $?$ & $?$ & 1 \\
\hline T.5 & & & & & & & & \\
\hline T.8 & & & & & & $?$ & $?$ & \\
\hline T.1 & & & & & $?$ & $?$ & $?$ & \\
\hline T.4 & & & & & & $?$ & $?$ & \\
\hline T.6 & & 2 & & & $?$ & $?$ & $?$ & 2 \\
\hline T.7 & & & & & $?$ & $?$ & $?$ & \\
\hline Total & & & & & 3 & & & \\
\hline
\end{tabular}

Tumuli type II

\begin{tabular}{l|c|c|c|c|c|c|c}
\hline Tum. & Shaft & Ch.1 & Ch.2 & Ch.3 & Ch.4 & Ch.5 & $=$ \\
\hline T.12 & & & & & & & \\
\hline T.13 & 1 & & & & & & 1 \\
\hline T.14 & & & & & & & \\
\hline T.15 & & & & & & & \\
\hline T.23 & & & & & & & \\
\hline T.24 & & & & & & & \\
\hline T.25 & 1 & & & & & & 1 \\
\hline T.26 & 3 & & & & & & \\
\hline T.16 & & & & & & & \\
\hline T.9 & & & & & & & \\
\hline T.1 & 1 & & & & & & \\
\hline Total & & & & & & & \\
\hline Vesstype & & & & & & & \\
\hline
\end{tabular}

Vessel type $\mathrm{XI}=9$

PRODUCED BY E. CZYŻEWSKA-ZALEWSKA

Only fragmented examples were found. More than 120 sherds were recorded, some of which could be pieced together and attributed to 23 vessels or groups of sherds (recorded in the database). The minimum number of ves- sels is nine. All sherds were found in the shafts and tunnels of type I and II tumuli, and most of them had been used as scrapers (see below, Chapter 5.2). 
1.11 GroupXII-Amphorae

TABLE 5.37 Amphorae of type XII.., distribution inside the tumuli

\begin{tabular}{l|l|l|l|l|l|l|l|l}
\hline \multicolumn{7}{l}{ Tumuli type I } \\
\hline Tum. & Shaft & Tun. & E Tun. & WTun. & Ch.1 & Ch.2 & Ch.3 & = \\
\hline T.2 & & & & & & & & \\
\hline T.3 & & & & & $?$ & $?$ & $?$ & \\
\hline T.5 & & & & & & & & \\
\hline T.8 & & & & & & $?$ & $?$ & \\
\hline T.1 & & & & & $?$ & $?$ & $?$ & \\
\hline T.4 & & & 2 & & & $?$ & $?$ & 2 \\
\hline T.6 & & & & & $?$ & $?$ & $?$ & \\
\hline T.7 & & 1 & & & $?$ & $?$ & $?$ & 1 \\
\hline Total & & & 3 & & & \\
\hline
\end{tabular}

Tumuli type II

\begin{tabular}{l|l|l|l|l|l|l|l}
\hline Tum. & Shaft & Ch.1 & Ch.2 & Ch.3 & Ch.4 & Ch.5 & $=$ \\
\hline T.12 & & & & & & & \\
\hline T.13 & & & & & & & \\
\hline T.14 & & & & & & & \\
\hline T.15 & & & & & & & \\
\hline T.23 & & & & & & & \\
\hline T.24 & 1 & & & & & & 1 \\
\hline T.25 & & & & & & & \\
\hline T.26 & & 1 & & & & & 1 \\
\hline T.16 & & & & & & & \\
\hline T.9 & & & & & & & \\
\hline T.11 & & & & & & & \\
\hline Total & & & & & & \\
\hline
\end{tabular}

Vessel type XII.1 $=5$

PRODUCED BY E. CZYŻEWSKA-ZALEWSKA 
TABLE 5.38 Amphorae of type XII.2, distribution inside the tumuli

\section{Tumuli type I}

\begin{tabular}{l|l|l|l|l|l|l|l|l}
\hline Tum. & Shaft & Tun. & E Tun. & WTun. & Ch.1 & Ch.2 & Ch.3 & $=$ \\
\hline T.2 & & & & & & & & \\
\hline T.3 & & & & & $?$ & $?$ & $?$ & \\
\hline T.5 & & & & & & & & \\
\hline T.8 & & & & & & $?$ & $?$ & \\
\hline T.1 & & & & & $?$ & $?$ & $?$ & \\
\hline T.4 & & & 1 & & & $?$ & $?$ & 1 \\
\hline T.6 & & & & & $?$ & $?$ & $?$ & \\
\hline T.7 & & & & & $?$ & $?$ & $?$ & \\
\hline Total & & & 1 & & & \\
\hline
\end{tabular}

Vessel type XII.2 = 1

TABLE 5.39 Amphorae of type XII.3, distribution inside the tumuli

\section{Tumuli type I}

\begin{tabular}{l|l|c|c|c|c|c|c|c}
\hline Tum. & Shaft & Tun. & E Tun. & WTun. & Ch.1 & Ch.2 & Ch.3 & $=$ \\
\hline T.2 & & & & & & & & \\
\hline T.3 & & & & & $?$ & $?$ & $?$ & \\
\hline T.5 & & & & & & & & \\
\hline T.8 & & & & & & $?$ & $?$ & \\
\hline T.1 & & & & & $?$ & $?$ & $?$ & \\
\hline T.4 & & & & & & $?$ & $?$ & \\
\hline T.6 & & & & & $?$ & $?$ & $?$ & \\
\hline T.7 & & sh & sh & sh & 1 & $?$ & $?$ & 3 \\
\hline Total & & & 3 & & & \\
\hline
\end{tabular}

Vessel type XII.3 = 3

PRODUCED BY E. CZYŻEWSKA-ZALEWSKA

Six types of amphorae were identified at the El-Zuma cemetery. There were five fragmented examples of type XII.1 Dongolan transport amphorae. Three examples came from type I tumuli (T.4 and T.7), where the sherds were recorded in the fill of the tunnels. Another two amphorae were found in tumuli of type II: one in the shaft of T.24, and another (the only amphora found in situ) in the main burial chamber of T.26. Some sherds of this type of amphora were used as scrapers. Imported amphorae from Asia
Minor (XII.2) are represented by only one example, found in the tunnel of T.4. This vessel was reused as a lamp (note that the other three oil lamps were also discovered in this tomb: see Chapter 3.6, this volume). Type XII.3, another type of transport amphora from Palestine, was represented by sherds found in the tunnels of T.7. Two vessels were restored from these sherds. It is highly probable that these amphorae were removed from the burial chamber, given that another broken amphora can be seen in photographs 
TABLE 5.40 Amphorae of type XII.4, distribution inside the tumuli

Tumuli type I

\begin{tabular}{l|l|l|l|l|l|l|l|l}
\hline Tum. & Shaft & Tun. & E Tun. & WTun. & Ch.1 & Ch.2 & Ch.3 & $=$ \\
\hline T.2 & & & & & & 1 & & 1 \\
\hline T.3 & & & & & $?$ & $?$ & $?$ & \\
\hline T.5 & & & & & & & & \\
\hline T.8 & & & & & & $?$ & $?$ & \\
\hline T.1 & & & & & $?$ & $?$ & $?$ & \\
\hline T.4 & & & & & & $?$ & $?$ & \\
\hline T.6 & & & & & $?$ & $?$ & $?$ & \\
\hline T.7 & & & & & $?$ & $?$ & $?$ & \\
\hline Total & & & & & & & & \\
\hline
\end{tabular}

\section{Tumuli type II}

\begin{tabular}{l|c|c|c|c|c|c|c}
\hline Tum. & Shaft & Ch.1 & Ch.2 & Ch.3 & Ch.4 & Ch.5 & $=$ \\
\hline T.12 & & & & & & & \\
\hline T.13 & & & & & & & \\
\hline T.14 & & & & & & & \\
\hline T.15 & 2 & 1 & & & & & 3 \\
\hline T.23 & & & & & & & \\
\hline T.24 & & & & & & & \\
\hline T.25 & & & & & & & \\
\hline T.26 & & & & & & & \\
\hline T.16 & & & & & & & \\
\hline T.9 & & & & & & & \\
\hline T.11 & & & & & & & \\
\hline Total & & & & 3 & & & \\
\hline
\end{tabular}

Vessel type XII.4 $=4$

PRODUCED BY E. CZYŻEWSKA-ZALEWSKA

taken of the unexcavated burial chamber of T.7 (see Volume I, Chapter 3, Fig. 3.41 bottom). Type XII.4 is a type of table amphora imported from the Aswan region. One example was found in chamber 2 of T.2 (type I), and three examples were discovered in T.15 (type II). Type XII.5 am- phorae are represented by one complete and one fragmented specimen recovered from two tumuli of type II. Only one amphora represents type XII.6 and was found, in pieces, in the shaft and tunnel of T.4. 
TABLE 5.41 Amphorae of type XII.5, distribution inside the tumuli

Tumuli type II

\begin{tabular}{l|l|l|l|l|l|l|l|l}
\hline Tum. & Shaft & Ch.1 & Ch.2 & Ch.3 & Ch.4 & Ch.5 & $=$ & $=$ \\
\hline T.12 & & & & & & & & \\
\hline T.13 & & & & & & & & \\
\hline T.14 & & & & & & & & \\
\hline T.15 & & & & & & & & \\
\hline T.23 & & & & & & & & \\
\hline T.24 & & & & & & & & \\
\hline T.25 & & & & 1 & & & & 1 \\
\hline T.26 & & & & & & & & \\
\hline T.16 & & 1 & & & & & & 1 \\
\hline T.9 & & & & & & & & \\
\hline T.11 & & & & & & & & \\
\hline Total & & & & & & & & \\
\hline
\end{tabular}

Vessel type XII.5 = 2

PRODUCED BY E. CZYŻEWSKA-ZALEWSKA

TABLE 5.42 Amphorae of type XII.6, distribution inside the tumuli

\section{Tumuli type I}

\begin{tabular}{l|c|c|c|c|c|c|c|c}
\hline Tum. & Shaft & Tun. & E Tun. & WTun. & Ch.1 & Ch.2 & Ch.3 & $=$ \\
\hline T.2 & & & & & & & & \\
\hline T.3 & & & & & $?$ & $?$ & $?$ & \\
\hline T.5 & & & & & & & & \\
\hline T.8 & & & & & & $?$ & $?$ & \\
\hline T.1 & & & & & $?$ & $?$ & $?$ & \\
\hline T.4 & 1 & & & & & $?$ & $?$ & 1 \\
\hline T.6 & & & & & $?$ & $?$ & $?$ & \\
\hline T.7 & & & & & $?$ & $?$ & $?$ & \\
\hline Total & & & & & & & \\
\hline
\end{tabular}

Vessel type XII. $6=1$

PRODUCED BY E. CZYŻEWSKA-ZALEWSKA 
1.12 GroupXIII-Bottles and XIV-Jugs

TABLE 5.43 Bottles of type XIII.1, distribution inside the tumuli

\begin{tabular}{|c|c|c|c|c|c|c|c|c|}
\hline \multicolumn{9}{|c|}{ Tumuli type I } \\
\hline Tum. & Shaft & Tun. & E Tun. & WTun. & Ch.1 & Ch.2 & Ch.3 & $=$ \\
\hline \multicolumn{9}{|l|}{ T.2 } \\
\hline T.3 & & & & & $?$ & $?$ & $?$ & \\
\hline \multicolumn{9}{|l|}{$\mathrm{T} .5$} \\
\hline T.8 & & & & & & $?$ & $?$ & \\
\hline T.1 & & & & & $?$ & $?$ & $?$ & \\
\hline T.4 & & & & & & $?$ & $?$ & \\
\hline T.6 & & 1 & & & $?$ & $?$ & $?$ & $\mathbf{1}$ \\
\hline $\mathrm{T} .7$ & & & & & $?$ & $?$ & $?$ & \\
\hline Total & & & & & & & & \\
\hline
\end{tabular}

Tumuli type II

\begin{tabular}{l|c|c|c|c|c|c|c}
\hline Tum. & Shaft & Ch.1 & Ch.2 & Ch.3 & Ch.4 & Ch.5 & $=$ \\
\hline T.12 & & & & & & & \\
\hline T.13 & 1 & & & & & & 1 \\
\hline T.14 & & & & & & & \\
\hline T.15 & & & & & & & \\
\hline T.23 & & & & & & & \\
\hline T.24 & & & & & & & \\
\hline T.25 & & & & & & & \\
\hline T.26 & & & & & & & \\
\hline T.16 & & & & & & & \\
\hline T.9 & & & & & & & \\
\hline T.11 & & & & & & & \\
\hline Total & & & & & & & \\
\hline
\end{tabular}

Vessel type XIII.1 = 2

PRODUCED BY E. CZYŻEWSKA-ZALEWSKA 
TABLE 5.44 Bottles of type XIII.2, distribution inside the tumuli

Tumuli type II

\begin{tabular}{l|l|l|l|l|l|l|l}
\hline Tum. & Shaft & Ch.1 & Ch.2 & Ch.3 & Ch.4 & Ch.5 & $=$ \\
\hline T.12 & & & & & & & \\
\hline T.13 & & & & & & & \\
\hline T.14 & & & & & & & \\
\hline T.15 & & & & & & & \\
\hline T.23 & & 1 & & & & & 1 \\
\hline T.24 & & & & & & & \\
\hline T.25 & & & & & & & \\
\hline T.26 & & & & & & & \\
\hline T.16 & & & & & & & \\
\hline T.9 & & & & & & & \\
\hline T.11 & & & & & & & \\
\hline Total & & & & & & \\
\hline
\end{tabular}

Vessel type XIII.2 $=1$

PRODUCED BY E. CZYŻEWSKA-ZALEWSKA

TABLE 5.45 Bottles of type XIII.3, distribution inside the tumuli

\section{Tumuli type III}

\begin{tabular}{l|l|l|l}
\hline Tum. & Shaft & Ch.1 & $=$ \\
\hline T.10 & & & \\
\hline T.17 & & & \\
\hline T.18 & & & \\
\hline T.19 & & & \\
\hline T.20 & & & \\
\hline T.22 & & & \\
\hline T.27 & & & \\
\hline T.21 & & 1 & 1 \\
\hline T.28 & & & \\
\hline Total & & 1 & \\
\hline
\end{tabular}

Vessel type XIII.3 = 1

PRODUCED BY E. CZYŻEWSKA-ZALEWSKA 
TABLE 5.46 Jugs of type XIV.1, distribution inside the tumuli

\begin{tabular}{l|l|l|l|l|l|l|l}
\hline \multicolumn{1}{l}{ Tumuli type II } & \multicolumn{5}{l}{} \\
\hline Tum. & Shaft & Ch.1 & Ch.2 & Ch.3 & Ch.4 & Ch.5 & $=$ \\
\hline T.12 & & & & & & & \\
\hline T.13 & & & & & & & \\
\hline T.14 & & & & & & & \\
\hline T.15 & 1 & & & & & & 1 \\
\hline T.23 & & & & & & & \\
\hline T.24 & & & & & & & \\
\hline T.25 & & & & & & & \\
\hline T.26 & & & & & & & \\
\hline T.16 & & & & & & & \\
\hline T.9 & & & & & & & \\
\hline T.11 & & & & & & & \\
\hline Total & & & & & & & \\
\hline
\end{tabular}

Vessel type XIV.1 = 1

PRODUCED BY E. CZYŻEWSKA-ZALEWSKA

TABLE 5.47 Jugs of type XIV.2, distribution inside the tumuli

\section{Tumuli type II}

\begin{tabular}{l|l|l|l|l|l|l|l}
\hline Tum. & Shaft & Ch.1 & Ch.2 & Ch.3 & Ch.4 & Ch.5 & $=$ \\
\hline T.12 & & & & & & & \\
\hline T.13 & & & & & & & \\
\hline T.14 & & & & & & & \\
\hline T.15 & & & 1 & & & & 1 \\
\hline T.23 & & & & & & & \\
\hline T.24 & & & & & & & \\
\hline T.25 & & & & & & & \\
\hline T.26 & & & & & & & \\
\hline T.16 & & & & & & & \\
\hline T.9 & & & & & & & \\
\hline T.11 & & & & & & & \\
\hline Total & & & & & & & \\
\hline Vesset & & & & & & \\
\hline
\end{tabular}

Vessel type XIV.2 $=1$

PRODUCED BY E. CZYŻEWSKA-ZALEWSKA 
Bottles in group XIII are represented by four bottles of three different types. One bottle of type XIII.1 was found in the tunnel of T.6 (type I) and another one was recovered from the shaft of T.13 (type II). A single bottle of type XIII.2 was discovered in the burial chamber of T.23, and one bottle of type XIII.3 was found in T.21 (type III). The small

2

\section{Distribution by Function}

The author tries to assign vessels to particular places inside the tumuli and determine the typical set of grave goods that was deposited in each type of tumulus.

The distribution of vessels presented below is based on the minimum number of vessels and encompasses all vessels attributed to specific groups, such as cups, bowls, or

\subsection{Group I, Cups}

Cups are widely represented in tumuli of type II: 77 of the 117 examples noted were recorded in this type of burial. Their occurrence in the chambers is varied. There is no specific pattern to their distribution. Various numbers were placed in the main burial chambers and additional chambers in almost all tumuli (except T.16). The greatest numbers of cups were found in T.12, T.14, T.23, and T.11. In the only two fully excavated type I tumuli (T.2 and T.5) several cups were found in different chambers, but there were number of vessels and type differentiation preclude any meaningful analysis of this group.

A similar situation exists with the group XIV jugs, of which there were only two, each representing a different type. Both were discovered in the same type II tumulus (T.15).

bottles, and includes vessels presented in the catalogue as varia. In the case of cups, bowls, goblets, and amphorae, objects photographed inside the unexcavated burial chambers of T.6 and T.7 (but not removed), are also included in this chapter.

also further examples in other graves of this type, where cups were found in the tunnels and external shafts. We cannot rule out that there may have been more of them in the burial chambers of these tumuli. The presence of cups was confirmed in the unexcavated burial chambers of T.6 and T.7 (see Volume I, Chapter 3, Fig. 3.35, Fig. 3.41 bottom). The situation with type II tombs is more straightforward, as cups were found in all graves of this type except T.17. 
TABLE 5.48 Group I, cups of all types, distribution inside the tumuli

\begin{tabular}{l|l|l|l|l|l|l|l|l|l}
\hline Tumuli type I \\
\hline Tum. & Mound & Shaft & Tun. & E Tun. & WTun. & Ch.1 & Ch.2 & Ch.3 & $=$ \\
\hline T.2 & & & & & & 2 & 2 & 4 \\
\hline T.3 & & & & & $?$ & $?$ & $?$ & \\
\hline T.5 & 5 & & & & & 1 & 1 & & 7 \\
\hline T.8 & & & & & & & $?$ & $?$ & \\
\hline T.1 & & & & & & $?$ & $?$ & $?$ & \\
\hline T.4 & & & 1 & 1 & 2 & & $?$ & $?$ & 4 \\
\hline T.6 & & 2 & & & & $3 ?$ & $?$ & $?$ & $2(5 ?)$ \\
\hline T.7 & 1 & 2 & & & & $1 ?$ & $?$ & $?$ & 4 \\
\hline Total & & & & & $23(24 ?)$ & & & & \\
\hline
\end{tabular}

\section{Tumuli type II}

\begin{tabular}{|c|c|c|c|c|c|c|c|}
\hline Tum. & Shaft & Ch.1 & Ch.2 & Ch.3 & Ch.4 & Ch.5 & $=$ \\
\hline T.12 & 1 & 1 & & 13 & & & 15 \\
\hline T.13 & & & 2 & & & & 2 \\
\hline T.14 & 1 & 5 & 2 & 3 & & & 11 \\
\hline T.15 & 4 & 2 & 2 & & & & 8 \\
\hline T.23 & 1 & 5 & 6 & & & & 12 \\
\hline T.24 & 1 & 2 & & 1 & & & 4 \\
\hline T.25 & & 1 & 1 & 5 & & & 7 \\
\hline T.26 & 1 & 7 & & & & & 8 \\
\hline \multicolumn{8}{|l|}{ T.16 } \\
\hline \multicolumn{8}{|l|}{ T.9 } \\
\hline T.11 & 1 & 5 & 4 & & & & 10 \\
\hline Total & & & & 77 & & & \\
\hline
\end{tabular}

Group I, cups $=117$

PRODUCED BY E. CZYŻEWSKA-ZALEWSKA

\section{Tumuli type III}

\begin{tabular}{|c|c|c|c|}
\hline Tum. & Shaft & Ch.1 & $=$ \\
\hline T.10 & & 7 & 7 \\
\hline \multicolumn{4}{|l|}{ T.17 } \\
\hline T.1 8 & & 1 & 1 \\
\hline T.19 & 1 & 1 & 2 \\
\hline T.2O & 1 & & $\mathbf{1}$ \\
\hline T.22 & & 2 & 2 \\
\hline T.27 & & 1 & 1 \\
\hline T.21 & 1 & & 1 \\
\hline T.28 & 1 & 3 & 4 \\
\hline Total & & 19 & \\
\hline
\end{tabular}


Group II, Small Bowls, Types II.1-II.7

TABLE 5.49 Group II, small bowls of types II.1-II.7, distribution inside the tumuli

Tumuli type I

\begin{tabular}{l|c|c|c|c|c|c|c|c|c}
\hline Tum. & Mound & Shaft & Tun. & E Tun. & WTun. & Ch.1 & Ch.2 & Ch.3 & $=$ \\
\hline T.2 & & & & & & & 20 & 7 & 27 \\
\hline T.3 & & 3 & 1 & & & $?$ & $?$ & $?$ & 4 \\
\hline T.5 & 2 & & 1 & & & & & & 3 \\
\hline T.8 & & & & & & 1 & $?$ & $?$ & 1 \\
\hline T.1 & & 2 & 1 & & & $?$ & $?$ & $?$ & 3 \\
\hline T.4 & & & 15 & 8 & 1 & $?$ & $?$ & $?$ & 24 \\
\hline T.6 & & 2 & 8 & 1 & 1 & $1 ?$ & $?$ & $?$ & 13 \\
\hline T.7 & 1 & & 2 & 8 & & $6 ?$ & $?$ & $?$ & 17 \\
\hline Total & & & & & 92 & & & & 13
\end{tabular}

Tumuli type II

\begin{tabular}{l|c|c|c|c|c|c|c}
\hline Tum. & Shaft & Ch.1 & Ch.2 & Ch.3 & Ch.4 & Ch.5 & $=$ \\
\hline T.12 & 28 & & 1 & 21 & & & 50 \\
\hline T.13 & 1 & & 2 & 5 & 4 & 2 & 14 \\
\hline T.14 & & 2 & 4 & 6 & & & 12 \\
\hline T.15 & 3 & 5 & 4 & & & & 12 \\
\hline T.23 & & 6 & 13 & & & & 19 \\
\hline T.24 & 1 & 4 & & & & & 5 \\
\hline T.25 & & & 5 & 3 & & & 8 \\
\hline T.26 & 1 & 4 & 20 & 1 & 4 & & 30 \\
\hline T.16 & & & & & & & \\
\hline T.9 & 3 & 1 & & & & & 4 \\
\hline T.11 & 1 & 4 & 10 & & & & 15 \\
\hline Total & & & & 169 & & & \\
\hline
\end{tabular}

Group II, small bowls of types II.1-II.7 $=276$

PRODUCED BY E. CZYŻEWSKA-ZALEWSKA
Tumuli type III

\begin{tabular}{l|c|c|c}
\hline Tum. & Shaft & Ch.1 & $=$ \\
\hline T.10 & & 11 & 11 \\
\hline T.17 & & & \\
\hline T.18 & & & \\
\hline T.19 & & 1 & 1 \\
\hline T.2O & & & \\
\hline T.22 & & & \\
\hline T.27 & & & \\
\hline T.21 & 2 & 1 & 3 \\
\hline T.28 & & & \\
\hline Total & & 15 & \\
\hline
\end{tabular}


Of the 276 small bowls noted, two-thirds (169 vessels) were recorded in tumuli of type II. Like cups, bowls were placed in different chambers but were present in greater numbers than cups and in the same type II tumuli as cups (except T.16). A large number of bowls was recorded in the fully excavated T.2 (27 vessels), but in all tumuli of this type bowls were found in the external shafts or in the tunnels. They were also noted in the unexplored chambers of T.6 and T.7 (see Volume I, Chapter 3, Fig. 3.35, Fig. 3.41 bottom). Bowls were recorded in only three of the smallest variety of tumuli (type III), the majority in the most richly furnished grave, T.10. Small bowls were less common than cups in tumuli of type III.

It is necessary to look at the cups and small bowls as a set of vessels that may have served a common function

\subsection{Group IV-VI, Bowls and Pots}

Pots of type VI are not represented among the grave goods; all examples were discovered in the shafts or the superstructure. But bowls and pots with an additional layer of clay added to the bottom (groups IV and v) were placed inside the burial chambers intentionally. Generally, they were abundant in type II burials. Fifty of the sixty recorded pots and bowls occurred in almost all tumuli of type II (except T.13). Most of these tumuli contained only a few vessels of this type, T.26 and T.11 featuring the greatest numbers. In type III burials, solitary examples of cooking vessels were recorded in only three tumuli.

If we look at the arrangement of grave goods inside the burial chambers, we can see that such vessels were in the graves. Large numbers of bowls were found inside the chambers together with animal bones. Most of the vessels were placed in groups, though in some cases they were spread out inside the chambers (see, for example, Chapter 7.19, Fig. 7.153, this volume). Sometimes a single cup was discovered among a set of bowls, but the number of bowls was always greater (see Volume I, Chapter 3, Fig. 3.7 bottom; Fig. 3.73 bottom; Fig. 3.63; Fig. 3.84; Fig. 105 bottom; Fig. 109 bottom).

The cups found in the burial chambers had another function, serving as lids for the large jars of group VII (see Volume I, Chapter 3, Fig. 3.7 top; Fig. 109 top; Fig. 3.117).

deposited together with the sets of bowls and cups described above (see Volume I, Chapter 3, Fig. 3.73; Fig. 3.117; Fig. 3.84; Fig. 3.89), and in the case of the group IV bowls, animal bones were also recorded inside these vessels (see Volume I, Chapter 8). An interesting example of the arrangement of these vessels was recorded in the atypical tumulus T.28 (see Volume I, Chapter 3), where a pot and bowls were deposited together with large jars in a niche, the bowl being positioned vertically. In this case, the bowl was not used as a container for a food offering, but seems to have been some kind of spare/supplementary vessel. 
TABLE 5.50 Groups IV-VI, bowls and pots, distribution inside the tumuli

Tumuli type I

\begin{tabular}{l|l|l|c|c|c|c|c|c|c}
\hline Tum. & Mound & Shaft & $\begin{array}{c}\text { Tun- } \\
\text { nel }\end{array}$ & $\begin{array}{c}\text { E } \\
\text { Tun. }\end{array}$ & $\begin{array}{c}\text { W } \\
\text { Tun. }\end{array}$ & Ch.1 & Ch.2 & Ch.3 & $=$ \\
\hline T.2 & & & & & & & & & \\
\hline T.3 & & & & & & $?$ & $?$ & $?$ & \\
\hline T.5 & & & 1 & & & & & & 1 \\
\hline T.8 & & & 1 & & & $?$ & $?$ & $?$ & 1 \\
\hline T.1 & & & & & & $?$ & $?$ & $?$ & \\
\hline T.4 & & & 1 & & & & $?$ & $?$ & 1 \\
\hline T.6 & & & & & & $?$ & $?$ & $?$ & \\
\hline T.7 & 1 & & 0.1 & & & $?$ & $?$ & $?$ & 1 \\
\hline Total & & & & & & & \\
\hline
\end{tabular}

Tumuli type II

\begin{tabular}{l|c|c|c|c|c|c|c|c}
\hline Tum. & Mound & Shaft & Ch.1 & Ch.2 & Ch.3 & Ch.4 & Ch.5 & $=$ \\
\hline T.12 & & & & & 2 & & & 2 \\
\hline T.13 & & & & & & & & \\
\hline T.14 & & & 1 & & & & & 1 \\
\hline T.15 & & & & & & & & \\
\hline T.23 & & 1 & & 1 & & & & 2 \\
\hline T.24 & & & 4 & & & & & 4 \\
\hline T.25 & & & & 4 & 4 & & & 8 \\
\hline T.26 & & 1 & 4 & 10 & 2 & 5 & 22 \\
\hline T.16 & & 1 & & & & & 1 \\
\hline T.9 & & & 1 & & & & & 1 \\
\hline T.11 & & 7 & 1 & 1 & & & & 9 \\
\hline Total & & & 50 & & \\
\hline
\end{tabular}

Groups IV-VI, bowls and pots $=60$

PRODUCED BY E. CZYŻEWSKA-ZALEWSKA
9

\section{Tumuli type III}

\begin{tabular}{l|c|c|c|c}
\hline Tum. & Mound & Shaft & Ch.1 & $=$ \\
\hline T.10 & & & 2 & 2 \\
\hline T.17 & & & & \\
\hline T.18 & & 1 & 1 & 2 \\
\hline T.19 & & & & \\
\hline T.20 & & & & \\
\hline T.22 & & & & \\
\hline T.27 & & & & \\
\hline T.21 & & & & \\
\hline T.28 & & \multicolumn{5}{|c}{6} \\
\hline Total & & 2 & 2 \\
\hline
\end{tabular}




\subsection{Group VII, Jars and Bottles}

TABLE 5.51 Group VII, jars, distribution inside the tumuli

\begin{tabular}{l|l|l|l|l|l|c|c|c|c}
\hline \multicolumn{1}{l}{ Tumuli type I } \\
\hline Tum. & Mound & Shaft & Tun. & E Tun. & WTun. & Ch.1 & Ch.2 & Ch.3 & $=$ \\
\hline T.2 & & & & & 1 & 3 & 3 & 7 \\
\hline T.3 & & & & & $?$ & $?$ & $?$ & \\
\hline T.5 & & 1 & & & & 1 & 1 & & 3 \\
\hline T.8 & & & & & & & $?$ & $?$ & \\
\hline T.1 & & & & & & $?$ & $?$ & $?$ & \\
\hline T.4 & & & & & & & $?$ & $?$ & \\
\hline T.6 & & & & 1 & & $2 ?$ & $?$ & $?$ & 3 \\
\hline T.7 & & & & & & $2 ?$ & $1 ?$ & $?$ & 3 \\
\hline Total & & & & & 16 & & & & \\
\hline
\end{tabular}

Tumuli type II

\begin{tabular}{|c|c|c|c|c|c|c|c|c|}
\hline Tum. & Mound & Shaft & Ch.1 & Ch.2 & Ch.3 & Ch.4 & Ch.5 & $=$ \\
\hline T.12 & & 19 & 8 & 4 & 3 & & & 34 \\
\hline T.13 & & 2 & & 3 & & & & 5 \\
\hline T.14 & 1 & 2 & & & 1 & & & 4 \\
\hline T.15 & & 1 & 5 & 2 & & & & 8 \\
\hline T.23 & & 1 & & & & & & $\mathbf{1}$ \\
\hline T.24 & & 3 & 2 & & 1 & 1 & & 7 \\
\hline T.25 & & & & 2 & & & & 2 \\
\hline T.26 & & 1 & & & 2 & & & 3 \\
\hline T.16 & & & 2 & & & & & 2 \\
\hline T.9 & & & & 2 & 1 & & & 3 \\
\hline T.11 & 2 & 2 & & 1 & & & & 5 \\
\hline Total & \multicolumn{8}{|c|}{74} \\
\hline
\end{tabular}

Group VII, jars and bottles $=114$

PRODUCED BY E. CZYŻEWSKA-ZALEWSKA

\begin{tabular}{|c|c|c|c|}
\hline \multicolumn{4}{|c|}{ Tumuli type II I } \\
\hline Tum. & Shaft & Ch.1 & $=$ \\
\hline T.10 & & 6 & 6 \\
\hline T.17 & & 2 & 2 \\
\hline T.18 & & 4 & 4 \\
\hline \multicolumn{4}{|l|}{ T.19 } \\
\hline T.2O & 1 & 3 & 4 \\
\hline T.22 & & 2 & 2 \\
\hline T.27 & & 1 & 1 \\
\hline T.21 & 1 & 1 & 2 \\
\hline $\mathrm{T} .28$ & 1 & 2 & 3 \\
\hline Total & & 24 & \\
\hline
\end{tabular}


Beer jars were recorded in all types of tumuli. Among the 225 recorded vessels or fragmented vessels, 113 were discovered complete, almost complete or in the form of diagnostic sherds, while the rest consisted of undiagnostic fragments or sherds used as scrapers. In the fully excavated type I tumuli, T.2 and T.5, several examples of jars were discovered in different chambers, and they were also recorded in the unexcavated chambers of T.6 and T.7. However, jars do not appear to have been abundant in these largest tumuli (see Volume I, Chapter 3, Fig. 3.35; Fig. 3.41). Like the cups and bowls discussed above, jars of group viI were abundant in type II graves and were discovered in all tumuli of this category. The richest in jars was T.12, where thirty-four of them were found. There is no order in the arrangement of jars. In four graves they were discovered in the main burial chamber, and in almost all tumuli they were found in additional chambers. T.23 was the only tomb in which jars were recovered from the shaft. Of the type III graves, T.19 was the only one in which a jar was not found. It seems that they were common in this type of grave, and together with cups they may have constituted a typical set of grave pottery deposited in the smallest burials.
In many graves they were placed inside the burial chamber against the walls, and often together in a group (see Volume I, Chapter 3, Fig. 3.117; Fig. 3.49 bottom; Fig. 3.63; Fig. 3.41). Some were deposited in the central part of the chamber, but also as part of a set of vessels (see Volume I, Chapter 3, T.2 Fig. 3.7 top; Fig. 3.48 bottom). In graves of type III, the fact that they were placed against the wall is no surprise given the small size of the burial chamber. Jars (as well as other vessels) were often put in the corners of the burial chamber, or positioned at the feet and head of the deceased (see Chapter 7.19, T.10 Fig. 7.142; T.27 Fig. 7.168, this volume). In some cases, robber activity had severely disturbed the original contexts, and it was impossible to reconstruct the burial arrangement (see Chapter 7.19, T.21 Fig. 7.169; T.19 Fig. 7.165, this volume). An interesting situation was observed in T.18, where the vessels were arranged in a row and occupied the entire length of the chamber (see Chapter 7.19, T.18 Fig. 7.164, this volume). 


\subsection{Group XII, Amphorae}

TABLE 5.52 Group XII, transport amphorae of types XII.1-XII.3, distribution inside the tumuli

\begin{tabular}{|c|c|c|c|c|c|c|c|c|}
\hline \multicolumn{9}{|c|}{ Tumuli type I } \\
\hline Tum. & Shaft & Tun. & E Tun. & WTun. & Ch.1 & Ch.2 & Ch.3 & $=$ \\
\hline \multicolumn{9}{|l|}{ T.2 } \\
\hline $\mathrm{T} .3$ & & & & & $?$ & $?$ & $?$ & \\
\hline \multicolumn{9}{|l|}{ T.5 } \\
\hline T.8 & & & & & & $?$ & $?$ & \\
\hline T.1 & & & & & $?$ & $?$ & $?$ & \\
\hline T.4 & & & 3 & & & $?$ & $?$ & 3 \\
\hline T.6 & & & & & $?$ & $?$ & $?$ & \\
\hline T.7 & & 1 & 1 & 1 & 1 & ? & ? & 4 \\
\hline Total & & & & & & & & \\
\hline
\end{tabular}

Tumuli type II

\begin{tabular}{l|c|c|c|c|c|c|c}
\hline Tum. & Shaft & Ch.1 & Ch.2 & Ch.3 & Ch.4 & Ch.5 & $=$ \\
\hline T.12 & & & & & & & \\
\hline T.13 & Sh. & & & & & & \\
\hline T.14 & 1 & & & & & & 1 \\
\hline T.15 & & & & & & & \\
\hline T.23 & & & & & & & \\
\hline T.24 & & & & & & & \\
\hline T.25 & & & & & & & \\
\hline T.26 & & 1 & & & & & \\
\hline T.16 & & & & & & & \\
\hline T.9 & & & & & & & \\
\hline T.11 & & & & & & & \\
\hline Total & & & & & & & \\
\hline
\end{tabular}

Group XII, transport amphorae, types XII.1-3 = 9

PRODUCED BY E. CZYŻEWSKA-ZALEWSKA 
TABLE 5.53 Group XII, table amphorae of types XII.4-XII.6, distribution inside the tumuli

\begin{tabular}{l|c|c|c|c|c|c|c|c}
\hline \multicolumn{7}{l}{ Tumuli type I } \\
\hline Tum. & Shaft & Tun. & E Tun. & WTun. & Ch.1 & Ch.2 & Ch.3 & $=$ \\
\hline T.2 & & & & & 1 & & 1 \\
\hline T.3 & 1 & & & & $?$ & $?$ & $?$ & 1 \\
\hline T.5 & & 1 & & & & & & 1 \\
\hline T.8 & & & & & & $?$ & $?$ & \\
\hline T.1 & & & & & $?$ & $?$ & $?$ & \\
\hline T.4 & 1 & 1 & & & & $?$ & $?$ & 2 \\
\hline T.6 & & & & & $?$ & $?$ & $?$ & \\
\hline T.7 & & & & & $?$ & $?$ & $?$ & \\
\hline Total & & & & 5 & & & & \\
\hline
\end{tabular}

\section{Tumuli type II}

\begin{tabular}{l|c|c|c|c|c|c|c}
\hline Tum. & Shaft & Ch.1 & Ch.2 & Ch.3 & Ch.4 & Ch.5 & $=$ \\
\hline T.12 & & & & & & & \\
\hline T.13 & & & & & & & \\
\hline T.14 & & & & & & & \\
\hline T.15 & 2 & 1 & & & & & 3 \\
\hline T.23 & & & & & & & \\
\hline T.24 & & & & & & & \\
\hline T.25 & & & 1 & & & & 1 \\
\hline T.26 & & & & & & & \\
\hline T.16 & 1 & & & & & & 1 \\
\hline T.9 & & & & & & & \\
\hline T.11 & & & & & & & \\
\hline Total & & & 5 & & & \\
\hline
\end{tabular}

Group XII, table amphorae, types XII.4-6 and varia $=10$

PRODUCED BY E. CZYŻEWSKA-ZALEWSKA 
Transport amphorae of types XII.1-XII.3 were not abundant at this site. Eleven amphorae were discovered in tombs of types I and II, but none were recorded in type III tumuli. The only amphora found in situ among the type I tumuli came from T.7 (see Volume I, Chapter 3, Fig. 3.41). T.26 (type II) was the only tomb in which a Dongolan amphora was found in situ in the main burial chamber, close to the remains of the deceased (see Chapter 7.3, Fig. 7.157, this volume).

\subsection{Other Vessels}

Other vessels, such as spouted bowls (group III), stands (group VIII), goblets (group IX), bottles (group XIII), and jugs (group XIV), were recorded in small numbers and only a few examples were discovered in situ (see above, Chapter 5.1). These vessels were deposited together with bowls and cups. The arrangement of the stand and bottle from T.23 could not be ascertained because they were placed in the main chamber, which was disturbed by looting,

\subsection{Scrapers}

Some vessels served a secondary function by being reused as scrapers. Among all of the recorded pottery there were 209 scrapers. The largest groups of vessels represented among the scrapers were jars and large bowls [Fig. 5.1].

Large bowls are broadly discussed in Chapter 5.1. The only vessel discovered in situ was the one in T.11. Most of the recorded sherds were used as scrapers. In Chapter 5.1 the minimum number of vessels is presented by the author. All bowls (except for the one found in T.11) were discovered in pieces, and among the 59 recorded sherds fortyone had been used as scrapers. Sherds were found in the external shafts and tunnels of type I tumuli, and in the shafts (predominantly) of type II tumuli. Several examples were also recovered from the shafts of type in tumuli.

The scrapers that made up the most significant group were made from jars of different types and were discovered in all types of tumuli, mainly in their shafts or tunnels, although a few examples were found in the chambers [Fig. 5.2].
Table amphorae of types XII.4-6 were recorded in the same types of tumuli. In T.2 one table amphora was discovered in situ together with a group of small bowls (see Volume I, Chapter 3, Fig. 3.7). Two amphorae were also found in situ in the burial chambers of T.15 and T.25, close to groups of small vessels such as cups or bowls.

but they were found in pieces together with bowls and cups (see Volume I, Chapter 3, Fig. 3.7 top). One goblet recorded in the burial chamber of T.1o was recorded in a group together with cups and bowls and animal bones (see Volume I, Chapter 3, T.10, Fig. 3.109 bottom). In T.24, a goblet was discovered inside the chamber together with spouted bowls and pots of group $\mathrm{V}$.

Twenty-five scrapers were made from qawadis. Among the 120 qadus sherds there were some broken scrapers that could be pieced together (see Chapter 4 , this volume). All sherds were discovered in the shafts or tunnels, and there is no evidence that these vessels had served as grave goods at the El-Zuma cemetery, despite the fact that the saqiya had been known in Ancient Nubia since Meroitic times (see Chapter 4, this volume).

Several sherds of transport amphorae were used as scrapers, but this was not a common function of this vessel type.

Scrapers were recorded in almost all tumuli at the ElZuma cemetery. Their function is obvious, but it was not possible to date their usage. Jars, qawadis, amphorae, and large bowls were known in Early Makurian times but are also noted at sites dating from the later period of the Makurian Kingdom (see Chapter 3.6, this volume). We should bear in mind that scrapers may have been used by tomb builders, as suggested by the scraper discovered

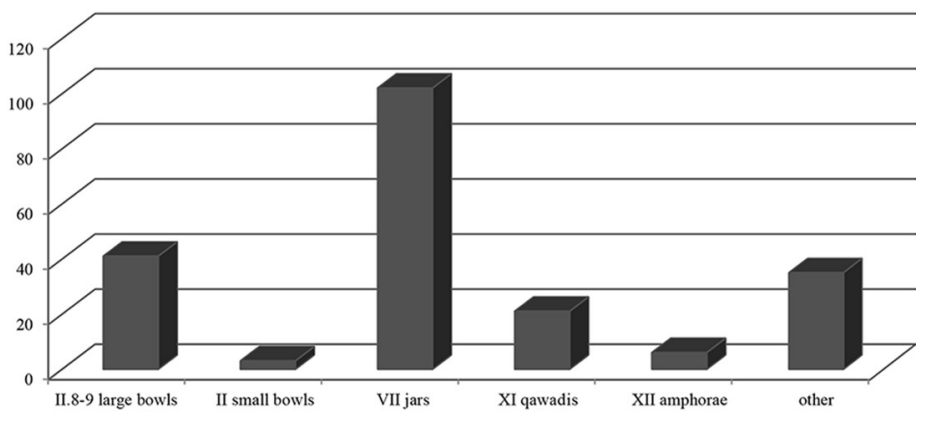

FIGURE 5.1

Occurrence of scrapers among various types of vessels PRODUCED BY E. CZYŻEWSKA-ZALEWSKA 


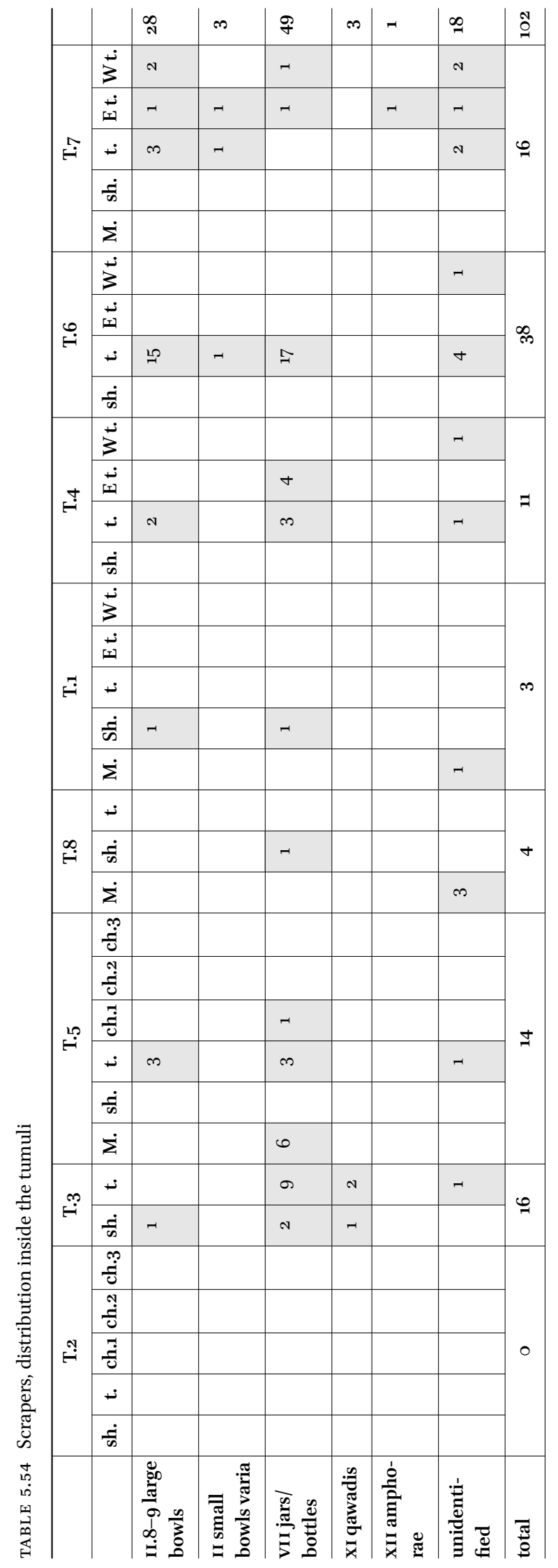




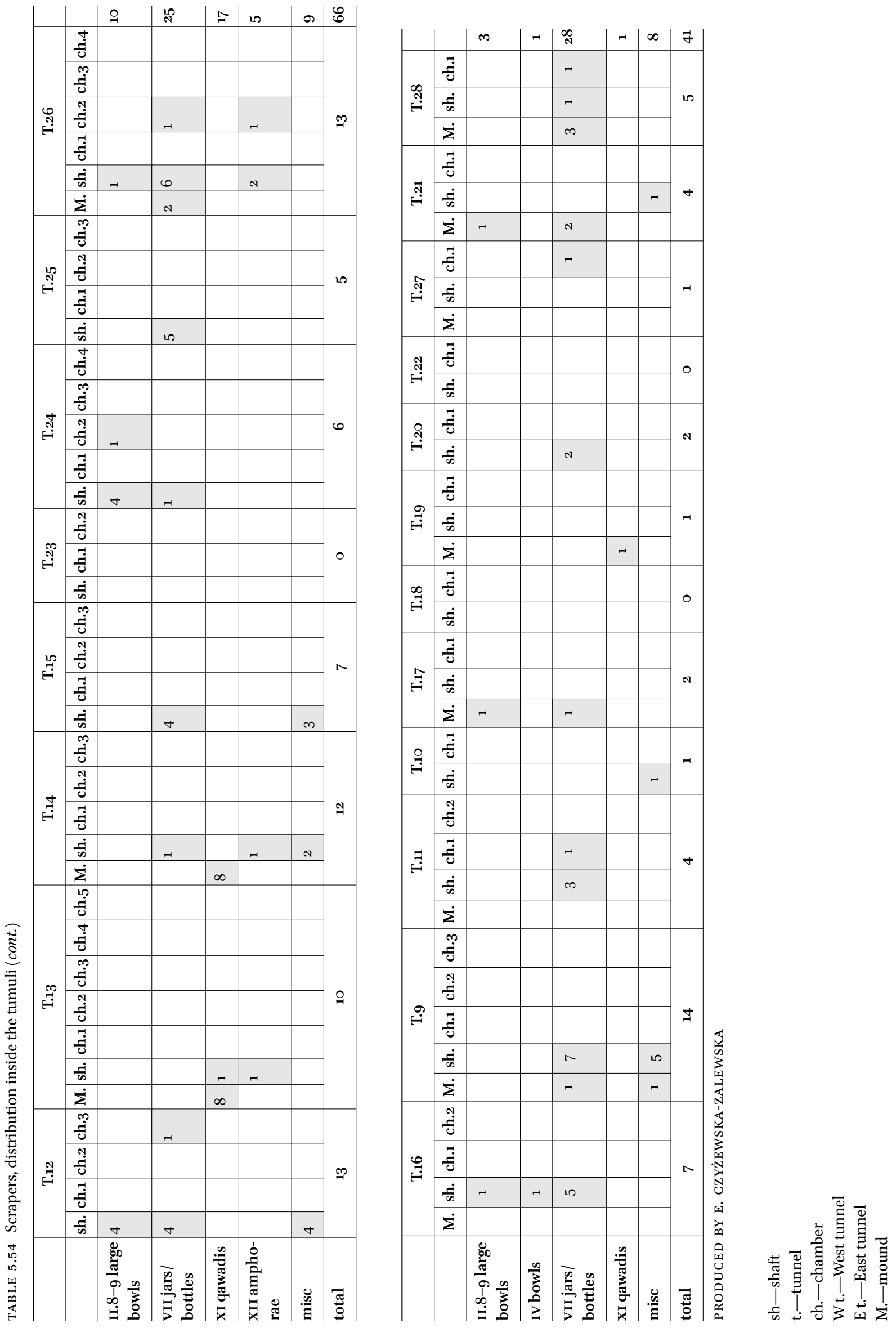




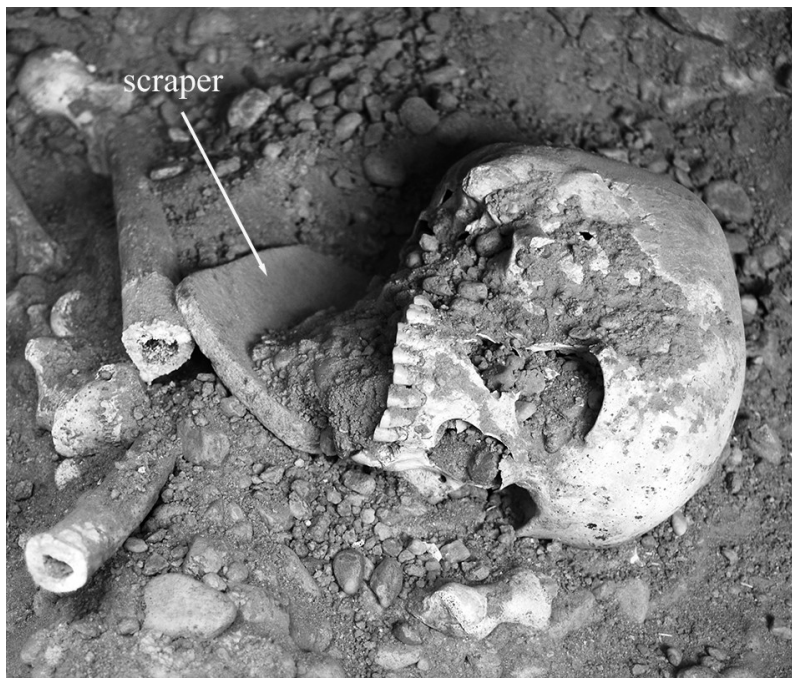

inside the intact chamber 3 in T.12 (see Volume I, Chapter 3.3). Another possibility is, of course, that they were used
FIGURE 5.2

A scraper found in the burial chamber of tumulus 17 PHOTO O. BIAŁOSTOCKA

\section{Discussion}

In the smallest type of burials (type III), which have only one chamber, we can observe that vessels were deposited mostly close to the head of the deceased and close to the legs. Such arrangements were recorded in T.28 (see Fig. 7.170), T.27 (see Fig. 7.168), T.17 (see Fig. 7.163), and T.10 (see Fig. 7.162). An interesting layout was noted in T.18 (see Fig. 7.164), where vessels were lined up alongside the chamber, which was quite narrow. Because of robber activity we cannot, of course, be sure if this was their original placement. In some tumuli, the original position was significantly disturbed, as in T.22 and T.19, where the bodies had been removed and left in the shaft together with burial equipment and vessels, in most cases broken (see Figs 7.165, 7.167).

In type II tumuli, which feature multiple chambers, the distribution of the vessels seems to be fairly random. In graves with two chambers, such as T.23 and T.11 (see Figs 7.153, 7.161), both chambers were abundantly equipped with pottery, and chamber 2 had more vessels than the main chamber, where the deceased was buried. In a few of the tumuli with three chambers, both of the addi-

by grave robbers. The majority of scrapers were discovered in the fills of shafts and tunnels.

tional chambers were provided with multiple vessels, as observed in T.25 (Fig. 7.156), T.14 (Fig. 7.150), and probably also T.12 (Fig. 7.144). In these instances, the pottery placed in the main chamber comprised a smaller assemblage than in the additional chambers. In tumuli with $4_{-5} 5$ chambers, we can see that the further from the main chamber, the fewer the number of grave goods. In T.26 (Fig. 7.157) and T.13 (Fig. 7.149) the largest quantity of pottery was found in chamber 2 , while chamber 5 contained only a few vessels. In both of these graves, the main chamber also held only a few vessels. A different situation was noted in T.24, where a sizeable collection was recorded in the main chamber, while the additional chambers contained only single vessels.

The distribution of grave goods in the chambers of type I tumuli is difficult to determine because only two of them were fully excavated: T.2 (see Fig. 7.135) and T.5 (Fig. 7.137). While a substantial amount of pottery was recorded in both additional chambers of T.2, which were entirely filled with grave goods, only several specimens were recorded in T.5. 